\title{
Optimal Dividend Strategies for Two Collaborating Insurance Companies
}

\author{
Hansjörg Albrecher, Pablo Azcue ${ }^{\dagger}$ and Nora Muler ${ }^{\dagger}$
}

\begin{abstract}
We consider a two-dimensional optimal dividend problem in the context of two insurance companies with compound Poisson surplus processes, who collaborate by paying each other's deficit when possible. We solve the stochastic control problem of maximizing the weighted sum of expected discounted dividend payments (among all admissible dividend strategies) until ruin of both companies, by extending results of univariate optimal control theory. In the case that the dividends paid by the two companies are equally weighted, the value function of this problem compares favorably with the one of merging the two companies completely. We identify this optimal value function as the smallest viscosity supersolution of the respective Hamilton-Jacobi-Bellman equation and provide an iterative approach to approximate it numerically. Curve strategies are identified as the natural analogue of barrier strategies in this two-dimensional context. A numerical example is given for which such a curve strategy is indeed optimal among all admissible dividend strategies, and for which this collaboration mechanism also outperforms the suitably weighted optimal dividend strategies of the two stand-alone companies.
\end{abstract}

\section{Introduction}

Ever since de Finetti [14] proposed in 1957 to measure the value of an insurance portfolio by the expected discounted sum of dividends paid during the lifetime of the portfolio, it has been of particular interest to determine the optimal dividend payment strategy which maximizes this quantity. More than that, this field of research over the years turned out to be a challenging and fascinating area, combining tools from analysis, probability and stochastic control. In 1969, Gerber [15] showed that if the free surplus of an insurance portfolio is modelled by a compound Poisson risk model, it is optimal to pay dividends according to a so-called band strategy, which collapses to a barrier strategy for exponentially distributed claim amounts. Whereas Gerber found this result by taking a limit of an associated discrete problem, this optimal dividend problem was studied with techniques of modern stochastic control theory in Azcue and Muler [7, see e.g. Schmidli 21] for a detailed overview. Since then the optimal dividend problem was studied for many different model setups, objective functions and side constraints (we refer to Albrecher and Thonhauser 2] and Avanzi [4] for surveys on the subject). A barrier strategy with barrier $b$ pays out dividends whenever the surplus level of the portfolio is above $b$, so that the surplus level stays at $b$, and pays no dividends below that barrier $b$. The most general criteria currently available for barrier strategies to be optimal can be found in Loeffen and Renaud 19. The optimality of barrier strategies when including the time value of ruin was studied in 23, and when including capital injections by shareholders

\footnotetext{
* Department of Actuarial Science, Faculty of Business and Economics, University of Lausanne, CH-1015 Lausanne and Swiss Finance Institute. Supported by the Swiss National Science Foundation Project 200020_143889.

${ }^{\dagger}$ Departamento de Matematicas, Universidad Torcuato Di Tella. Av. Figueroa Alcorta 7350 (C1428BIJ) Ciudad de Buenos Aires, Argentina.
} 
in Kulenko and Schmidli [18].

All these control problems have been formulated and studied in the one-dimensional framework. However, in recent years there has been an increased interest in risk theory in considering the dynamics of several connected insurance portfolios simultaneously, see e.g. Asmussen and Albrecher [3, Ch.XIII.9] for an overview. Ruin probability expressions for a two-dimensional risk process are studied in Avram et al. [5, 6] for simultaneous claim arrivals and proportional claim sizes and recently in Badila et al. [11 and Ivanovs and Boxma 17] in a more general framework. In Azcue and Muler [8, the problem of optimally transferring capital between two portfolios in the presence of transaction costs was considered, see also Badescu et al. [10. Czarna and Palmowski [13] study the dividend problem and impulse control for two insurance companies who share claim payments and premiums in some specified proportion for a particular dividend strategy. It turns out that these multi-dimensional problems, albeit practically highly relevant, quickly become very intricate and explicit solutions can typically not be obtained without very strong assumptions.

In this paper, we would like to extend the optimal dividend problem from univariate risk theory to a two-dimensional setup of two collaborating companies. The collaboration consists of paying the deficit ('bailing out') of the partner company if its surplus is negative and if this financial help can be afforded with the current own surplus level. We solve the problem of maximizing the weighted sum of expected discounted dividend payments until ruin of both companies. A natural question in this context is whether such a collaboration procedure can be advantageous over merging the two companies completely; we will show that this is the case when the dividends paid by the two companies are equally weighted. For criteria of a merger being an advantage over keeping two stand-alone companies under pre-defined barrier strategies and marginal diffusion processes, see e.g. Gerber and Shiu [16], for the performance of another pre-defined risk and profit sharing arrangement, see e.g. Albrecher and Lautscham [1]. Our goal here is, however, to address the general problem of identifying the optimal dividend strategy (among all admissible dividend strategies) for each company under this collaboration framework. This leads to a fully two-dimensional stochastic control problem, and to the question what the natural analogues of the optimal univariate barrier strategies are in two dimensions. The particular structure of the collaboration implemented in this paper will turn out not to be essential, so the techniques may be applicable to other risk-sharing mechanisms as well. Yet, the concrete specification allows to carry through the necessary analysis of the stochastic control problem explicitly by way of example.

The rest of the paper is organized as follows. In Section 2 we introduce the model and the stochastic control problem in detail and derive some simple properties of the corresponding value function $V$. In Section 3 we prove that $V$ is a viscosity solution of the corresponding Hamilton-Jacobi-Bellman equation for independent surplus processes, and in Section 4 we show that $V$ is in fact its smallest viscosity supersolution. Section 5 provides an iterative approach to approximate the value function $V$, together with the analogous verification steps at each iteration step. Section [6 discusses the stationary dividend strategies that appear in our model, and in Section 7 we establish curve strategies as the appropriate analogues of the univariate barrier strategies. Finally, Section 8 shows how to constructively search for optimal curve strategies and in Section 9 an explicit numerical example for the symmetric (and equally weighted) case with exponentially distributed claim sizes is worked out for which such a curve strategy is indeed optimal among all admissible bivariate dividend strategies. It is then also illustrated that for this case the proposed type of collaboration is preferable to adding the best-possible stand-alone profits. 


\section{Model}

We consider two insurance companies, Company One and Company Two, which have an agreement to collaborate. Let us call $X_{t}$ the free surplus of Company One and $Y_{t}$ the one of Company Two. We assume that the free surplus of each of the companies follows a CramérLundberg process, i.e. a compound Poisson process with drift given by

$$
\left\{\begin{array}{l}
X_{t}=x+p_{1} t-\sum_{i=1}^{N_{t}^{1}} U_{i}^{(1)} \\
Y_{t}=y+p_{2} t-\sum_{i=1}^{N_{t}^{2}} U_{i}^{(2)},
\end{array}\right.
$$

where $x$ and $y$ are the respective initial surplus levels; $p_{1}$ and $p_{2}$ are the respective premium rates; $U_{i}^{(k)}$ is the size of the $i$-th claim of Company $k$, which are i.i.d. random variables with continuous distribution $F^{k}$ for $k=1,2 ; N_{t}^{1}$ and $N_{t}^{2}$ are Poisson processes with intensity $\lambda_{1}$ and $\lambda_{2}$, respectively. We assume here that the processes $N_{t}^{1}, N_{t}^{2}$ and the random variables $U_{i}^{(1)}$, $U_{i}^{(2)}$ are all independent of each other, and $p_{j}>\lambda_{j} E\left(U_{i}^{(j)}\right), j=1,2$.

There is a rule of collaboration signed by the two companies: if the current surplus of Company One becomes negative, Company Two should cover the exact deficit of Company One as long as it does not ruin itself, and vice versa. Ruin of a company hence occurs when its surplus becomes negative and the other company cannot cover this deficit.

A simulated surplus trajectory under this collaboration rule is shown in Figure 1.1.

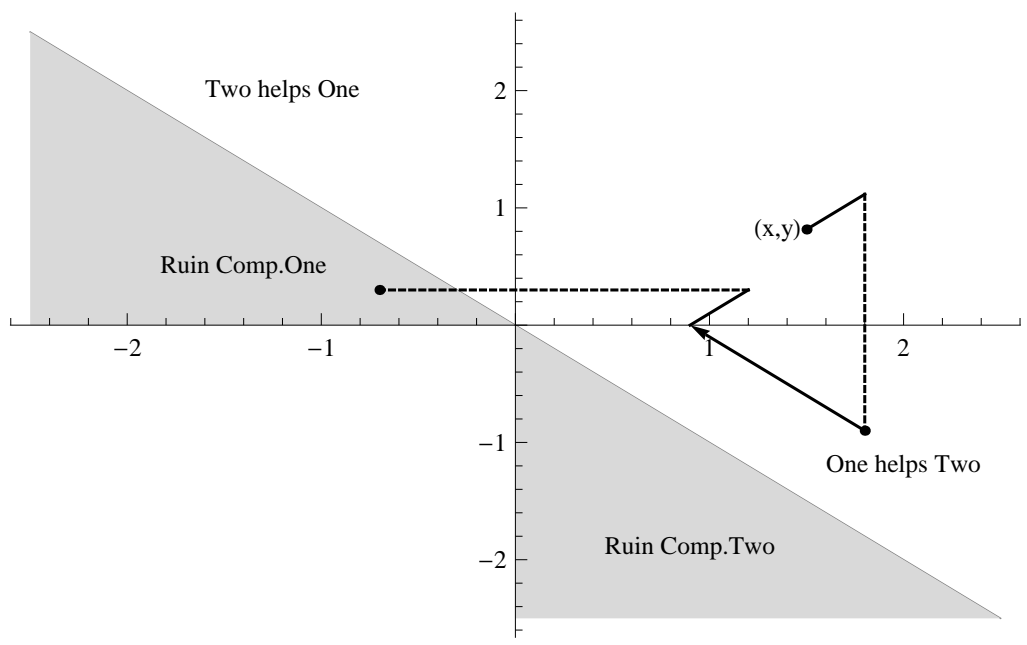

Fig. 1.1: Surplus process under the collaboration rules.

Both companies use part of their surplus to pay dividends to their shareholders. The dividend payment strategy $\bar{L}=\left(L_{t}^{1}, L_{t}^{2}\right)$ is the total amount of dividends paid by the two companies up to time $t$. Let us call $\tau_{i}^{k}$ the arrival time of the $i$-th claim of company $k$, with $k=1,2$. We define the associated controlled process $\left(X_{t}^{\bar{L}}, Y_{t}^{\bar{L}}\right)$ with initial surplus levels $(x, y)$ as

$$
\left\{\begin{array}{l}
X_{t}^{\bar{L}}=X_{t}-L_{t}^{1}+C_{t}^{2,1}-C_{t}^{1,2} \\
Y_{t}^{\bar{L}}=Y_{t}-L_{t}^{2}+C_{t}^{1,2}-C_{t}^{2,1}
\end{array}\right.
$$

where 


$$
C_{t}^{2,1}=\sum_{i=1}^{N_{t}^{1}} I X_{\left.\tau_{i}^{1}<0, Y_{\tau_{i}^{1}}^{\bar{L}}+X_{\tau_{i}^{1}}^{\bar{L}} \geq 0\right\}}\left|X_{\tau_{i}^{1}}^{\bar{L}}\right|
$$

corresponds to the cumulative amount transferred from Company Two to Company One up to time $t$ in order to cover the deficit of Company One and

$$
C_{t}^{1,2}=\sum_{i=1}^{N_{t}^{2}} I\left\{Y_{\left.\tau_{i}^{2}<0, Y_{\tau_{i}^{2}}^{\bar{L}}+X_{\tau_{i}^{2}}^{\bar{L}} \geq 0\right\}}\left|Y_{\tau_{i}^{2}}^{\bar{L}}\right|\right.
$$

corresponds to the cumulative amount transferred from Company One to Company Two up to time $t$ in order to cover the deficit of Company Two.

Let us call $\bar{\tau}$ the time at which only one company remains (because it cannot cover the deficit of the other), more precisely,

$$
\bar{\tau}=\inf \left\{t \geq 0: X_{t}^{\bar{L}}+Y_{t}^{\bar{L}}<0\right\}
$$

The process $\left(X_{t}^{\bar{L}}, Y_{t}^{\bar{L}}\right)$ is defined for $t \leq \bar{\tau}$. We say that the dividend payment strategy $\bar{L}=\left(L_{t}^{1}, L_{t}^{2}\right)_{t \leq \bar{\tau}}$ is admissible if it is non-decreasing, càglàd (left continuous with right limits), predictable with respect to the filtration generated by the bivariate process $\left(X_{t}, Y_{t}\right)$, and satisfies

$$
\left\{\begin{array}{c}
L_{t}^{1} \leq X_{t}+C_{t}^{2,1}-C_{t}^{1,2} \\
L_{t}^{2} \leq Y_{t}+C_{t}^{1,2}-C_{t}^{2,1}
\end{array}\right.
$$

This last condition means that the companies are not allowed to pay more dividends than their current surplus. Let us call $\mathbf{R}_{+}^{2}$ the first quadrant. We denote by $\Pi_{x, y}$ the set of admissible dividend strategies with initial surplus levels $(x, y) \in \mathbf{R}_{+}^{2}$. Our objective is to maximize the weighted average of the expected discounted dividends paid by the two companies until ruin of both companies. Note that after time $\bar{\tau}$, the surviving company can continue to pay dividends up to its own ruin. Let us define $V_{k}^{0}(k=1,2)$ as the optimal value function of the onedimensional problem of maximizing the expected discounted dividends until ruin of Company $k$ alone. So, for any initial surplus levels $(x, y) \in \mathbf{R}_{+}^{2}$, we can write down the optimal value function as

$$
V(x, y)=\sup _{\bar{L} \in \Pi_{x, y}} V_{\bar{L}}(x, y)
$$

where

$$
V_{\bar{L}}(x, y)=E_{x, y}\left(a_{1}\left(\int_{0}^{\bar{\tau}} e^{-\delta s} d L_{s}^{1}+e^{-\delta \bar{\tau}} V_{1}^{0}\left(X_{\bar{\tau}}^{\bar{L}}\right)\right)+a_{2}\left(\int_{0}^{\bar{\tau}} e^{-\delta s} d L_{s}^{2}+e^{-\delta \bar{\tau}} V_{2}^{0}\left(Y_{\bar{\tau}}^{\bar{L}}\right)\right)\right)
$$

Here, $\delta>0$ is a constant discount factor, and $a_{1} \in[0,1]$ and $a_{2}=1-a_{1}$ are the weights of the dividends paid by Company One and Company Two respectively. The functions $V_{k}^{0}(k=1,2)$ are zero in $(-\infty, 0)$ so depending on which company goes to ruin at $\bar{\tau}$, either $V_{1}^{0}\left(X_{\bar{\tau}}\right)=0$ or $V_{2}^{0}\left(Y_{\bar{\tau}}\right)=0$. The optimal dividend strategy corresponding to (4) may be regarded as the best dividend payment strategy from the point of view of a shareholder who owns a proportion $m a_{1}$ of the total shares of Company One and a proportion $m a_{2}$ of the total shares of Company Two for some $0<m \leq \min \left\{1 / a_{1}, 1 / a_{2}\right\}$. An important particular case is $a_{1}=a_{2}=1 / 2$, in which the dividends paid by the two companies are equally weighted (for an earlier example of weighting separate terms in the objective function in the univariate dividend context, see Radner and Shepp [20]). 
Remark 1 In case the two companies are owned by the same shareholders, another possibility of collaboration between the two companies is merging, in which case the companies put together all their surplus, pay the claims of both companies and pay dividends up to time $\tau$ at which the joined surplus becomes negative (see e.g. Gerber and Shiu [16])). Given the initial surplus levels $(x, y)$, we can interpret any admissible dividend payment strategy $\left(L_{t}\right)_{t \geq 0}$ for the merger as an admissible collaborating one as follows

$$
L_{t}^{1}=\int_{0}^{t} \frac{X_{t}^{\bar{L}}}{X_{t}^{\bar{L}}+Y_{t}^{\bar{L}}} d L_{t}, L_{t}^{2}=\int_{0}^{t} \frac{Y_{t}^{\bar{L}}}{X_{t}^{\bar{L}}+Y_{t}^{\bar{L}}} d L_{t}
$$

Since $L_{t}$ is constant for $t \geq \tau=\bar{\tau}$, the surviving company does not pay any dividends here. So $V_{\bar{L}}$ defined in (5) for $a_{1}=a_{2}=1 / 2$, satisfies

$$
2 V_{\bar{L}}(x, y)>2 E_{x, y}\left(\frac{1}{2} \int_{0}^{\bar{\tau}} e^{-\delta s} d L_{s}^{1}+\frac{1}{2} \int_{0}^{\bar{\tau}} e^{-\delta s} d L_{s}^{2}\right)=E_{x+y}\left(\int_{0}^{\tau} e^{-\delta s} d L_{s}\right)
$$

The last expected value is the value function of the merger dividend strategy $\left(L_{t}\right)_{t>0}$. We conclude that the optimal collaborating strategy for equally weighted dividend payments is better than the optimal merger strategy.

Both optimal value functions $V_{1}^{0}$ and $V_{2}^{0}$ corresponding to the stand-alone companies have an ultimately linear growth with slope one and they are Lipschitz, see for instance Azcue and Muler [7]. Let us state some basic results about regularity and growth at infinity of the optimal value function $V$ defined in (4). From now on, let us call $\lambda:=\lambda_{1}+\lambda_{2}$ and $p:=a_{1} p_{1}+a_{2} p_{2}$.

Lemma 2 The optimal value function is well defined and satisfies

$$
a_{1} x+a_{2} y+\frac{p}{\delta+\lambda} \leq V(x, y) \leq a_{1} x+a_{2} y+\frac{p}{\delta}
$$

for all $(x, y) \in \mathbf{R}_{+}^{2}$.

Proof.

Let us first prove the second inequality. Note that $V(x, y)$ increases when the Poisson intensities $\lambda_{1}$ and $\lambda_{2}$ decrease, but the optimal value function for the problem with parameters $\lambda_{1}=\lambda_{2}=0$ is

$$
a_{1} x+a_{2} y+\int_{0}^{\infty} e^{-\delta s} p d s=a_{1} x+a_{2} y+\frac{p}{\delta},
$$

which corresponds to the value function of the strategy in which each company pays immediately the initial surplus and then it pays the incoming premium forever as dividends.

In order to obtain the first inequality, consider the admissible strategy $\bar{L}_{0}=\left(L_{t}^{1}, L_{t}^{2}\right)$ in which each company pays the initial surplus immediately, and then pays the incoming premium up to time $\bar{\tau}$ that coincides with the first claim arrival time $\tau_{1}=\tau_{1}^{1} \wedge \tau_{1}^{2}$; we have,

$$
\begin{aligned}
V(x, y) & \geq V_{\bar{L}_{0}}(x, y) \\
& \geq a_{1} x+a_{2} y+E_{x, y}\left(\int_{0}^{\tau_{1}} e^{-\delta s} p d s\right) \\
& =a_{1} x+a_{2} y+\frac{p}{\delta+\lambda} .
\end{aligned}
$$

Lemma 3 The optimal value function $V$ is increasing, locally Lipschitz and satisfies for any $(x, y) \in \mathbf{R}_{+}^{2}$,

$$
a_{1} h \leq V(x+h, y)-V(x, y) \leq\left(e^{(\delta+\lambda) h / p_{1}}-1\right) V(x, y)
$$

and

$$
a_{2} h \leq V(x, y+h)-V(x, y) \leq\left(e^{(\delta+\lambda) h / p_{2}}-1\right) V(x, y)
$$

for any $h>0$. 
Proof.

Let us prove the inequalities at the top, the ones at the bottom are similar. Given any $\varepsilon>0$, take an admissible strategy $\bar{L} \in \Pi_{x, y}$ such that $V_{\bar{L}}(x, y) \geq V(x, y)-\varepsilon$. We define the strategy $\bar{L}^{1} \in \Pi_{x+h, y}$ for $h>0$ as follows: Pay immediately an amount $h$ of the surplus of Company One as dividends and then follow the strategy $\bar{L}$. We have that

$$
V_{\bar{L}^{1}}(x+h, y)=V_{\bar{L}}(x, y)+a_{1} h
$$

and so

$$
V(x+h, y) \geq V_{\bar{L}}(x, y)+a_{1} h>V(x, y)+a_{1} h-\varepsilon .
$$

Consider also an admissible strategy $\bar{L}^{2} \in \Pi_{x+h, y}$ such that $V(x+h, y) \geq V_{\bar{L}^{2}}(x+h, y)-\varepsilon$ and define the admissible strategy $\bar{L}^{3} \in \Pi_{x, y}$ which, starting with surplus $(x, y)$ pays no dividends until

$$
\tilde{\tau}=\inf \left\{t \geq 0: X_{t}^{\bar{L}^{3}} \geq x+h, Y_{t}^{\bar{L}^{3}} \geq y\right\},
$$

at time $\tilde{\tau}$ pays either $X_{t}^{\bar{L}^{3}}-(x+h)$ from the surplus of Company One or $Y_{t}^{\bar{L}^{3}}-y$ from the surplus of Company Two, depending on which of these differences is positive, and then follows strategy $\bar{L}^{2} \in \Pi_{x+h, y}$. In the event of no claims, $\tilde{\tau}=t_{0}:=h / p_{1}$; since the probability of no claims until $t_{0}$ is $e^{-\lambda t_{0}}$, we get

$$
V(x, y) \geq V_{\bar{L}^{3}}(x, y) \geq V_{\bar{L}^{2}}(x+h, y) e^{-(\delta+\lambda) t_{0}} \geq(V(x+h, y)-\varepsilon) e^{-(\delta+\lambda) t_{0}} .
$$

From (6) and (7), we get the inequalities at the top.

\section{Hamilton-Jacobi-Bellman equation}

In order to obtain the Hamilton-Jacobi-Bellman (HJB) equation associated to the optimization problem (4), we need to state the so called Dynamic Programming Principle (DPP). The proof that this holds is similar to the one given in Lemma 1.2 of Azcue and Muler [9] and uses that $V$ is increasing and continuous in $\mathbf{R}_{+}^{2}$.

Lemma 4 For any initial surplus $(x, y)$ in $\mathbf{R}_{+}^{2}$ and any stopping time $\tau$, we can write

$$
\begin{aligned}
& V(x, y) \\
& =\sup _{\bar{L} \in \Pi_{x, y}}\left(E _ { x , y } \left(a_{1} \int_{0}^{\tau \wedge \bar{\tau}} e^{-\delta s} d L_{s}^{1}+a_{2} \int_{0}^{\tau \wedge \bar{\tau}} e^{-\delta s} d L_{s}^{2}+e^{-\delta(\tau \wedge \bar{\tau})} I_{\{\tau \wedge \bar{\tau}<\bar{\tau}\}} V\left(X_{\tau \wedge \bar{\tau}}^{\bar{L}}, Y_{\tau \wedge \bar{\tau}}^{\bar{L}}\right)\right.\right. \\
& \left.\left.\quad+e^{-\delta(\tau \wedge \bar{\tau})} I_{\{\tau \wedge \bar{\tau}=\bar{\tau}\}}\left(a_{1} V_{1}^{0}\left(X_{\bar{\tau}}^{\bar{L}}\right)+a_{2} V_{2}^{0}\left(Y_{\bar{\tau}}^{\bar{L}}\right)\right)\right)\right) .
\end{aligned}
$$

The HJB equation of this optimization problem is

$$
\max \left\{\mathcal{L}(V)(x, y), a_{1}-V_{x}(x, y), a_{2}-V_{y}(x, y)\right\}=0
$$

where

$$
\begin{gathered}
\mathcal{L}(V)(x, y)=V_{x}(x, y) p_{1}+V_{y}(x, y) p_{2}-(\delta+\lambda) V(x, y)+\mathcal{I}(V)(x, y)+U(x, y), \\
\mathcal{I}(V)(x, y)=\begin{array}{c}
\lambda_{1} \int_{0}^{x} V(x-\alpha, y) d F^{1}(\alpha)+\lambda_{1} \int_{x}^{x+y} V(0, x+y-\alpha) d F^{1}(\alpha) \\
+ \\
\quad \lambda_{2} \int_{0}^{y} V(x, y-\alpha) d F^{2}(\alpha)+\lambda_{2} \int_{y}^{x+y} V(x+y-\alpha, 0) d F^{2}(\alpha),
\end{array}
\end{gathered}
$$


and

$$
U(x, y)=\lambda_{1} a_{2} V_{2}^{0}(y)\left(1-F^{1}(x+y)\right)+\lambda_{2} a_{1} V_{1}^{0}(x)\left(1-F^{2}(x+y)\right) .
$$

Since the optimal value function $V$ is locally Lipschitz but possibly not differentiable at certain points, we cannot say that $V$ is a solution of the HJB equation, we prove instead that $V$ is a viscosity solution of the corresponding HJB equation. Let us define this notion (see Crandell and Lions 12 and Soner 22 for further details).

Definition 5 A locally Lipschitz function $\bar{u}: \mathbf{R}_{+}^{2} \rightarrow \mathbf{R}$ is a viscosity supersolution of (8) at $(x, y) \in \mathbf{R}_{+}^{2}$ if any continuously differentiable function $\varphi: \mathbf{R}_{+}^{2} \rightarrow \mathbf{R}$ with $\varphi(x, y)=\bar{u}(x, y)$ such that $\bar{u}-\varphi$ reaches the minimum at $(x, y)$ satisfies

$$
\max \left\{\mathcal{L}(\varphi)(x, y), a_{1}-\varphi_{x}(x, y), a_{2}-\varphi_{y}(x, y)\right\} \leq 0 .
$$

A function $\underline{u}: \mathbf{R}_{+}^{2} \rightarrow \mathbf{R}$ is a viscosity subsolution of (8) at $(x, y) \in \mathbf{R}_{+}^{2}$ if any continuously differentiable function $\psi: \mathbf{R}_{+}^{2} \rightarrow \mathbf{R}$ with $\psi(x, y)=\underline{u}(x, y)$ such that $\underline{u}-\psi$ reaches the maximum at $(x, y)$ satisfies

$$
\max \left\{\mathcal{L}(\psi)(x, y), a_{1}-\psi_{x}(x, y), a_{2}-\psi_{y}(x, y)\right\} \geq 0 .
$$

A function $u: \mathbf{R}_{+}^{2} \rightarrow \mathbf{R}$ which is both a supersolution and subsolution at $(x, y) \in \mathbf{R}_{+}^{2}$ is called a viscosity solution of (8) at $(x, y) \in \mathbf{R}_{+}^{2}$.

Proposition $6 V$ is a viscosity supersolution of the HJB equation (8) at any $(x, y)$ with $x>0$ and $y>0$.

Proof. Given initial surplus levels $x>0, y>0$ and any $l_{1} \geq 0, l_{2} \geq 0$, let us consider the admissible strategy $\bar{L}$ where Company One and Two pay dividends with constant rates $l_{1}$ and $l_{2}$ respectively and $\bar{\tau}$ is defined as in (3). Let $\varphi$ be a test function for the supersolution of (8) at $(x, y)$ with $x>0$ and $y>0$. As before, denote $\tau_{1}^{1}$ and $\tau_{1}^{2}$ as the arrival time of the first claim of Company One and Two respectively, and $\tau_{1}=\tau_{1}^{1} \wedge \tau_{1}^{2}$. We have for $t<\tau_{1}$,

$$
\left\{\begin{array}{l}
X_{t}^{\bar{L}}=x+\left(p_{1}-l_{1}\right) t, \\
Y_{t}^{\bar{L}}=y+\left(p_{2}-l_{2}\right) t .
\end{array}\right.
$$

Note that $N_{t}^{1}+N_{t}^{2}$ is a Poisson process with intensity $\lambda$, because the arrival times of the two companies are independent. We have from Lemma 4 that

$$
\begin{aligned}
\varphi(x, y)= & V(x, y) \\
\geq & E_{x, y}\left(a_{1} \int_{0}^{\tau_{1} \wedge t} e^{-\delta s} l_{1} d s+a_{2} \int_{0}^{\tau_{1} \wedge t} e^{-\delta s} l_{2} d s\right) \\
& \left.+E_{x, y}\left(e^{-\delta\left(\tau_{1} \wedge t\right)} I_{\left\{\tau_{1} \wedge t<\bar{\tau}\right\}} V\left(X_{\tau_{1} \wedge t}^{\bar{L}}, Y_{\tau_{1} \wedge t}^{\bar{L}}\right)\right)\right) \\
& +E_{x, y}\left(e^{-\delta \bar{\tau}} I_{\left\{\tau_{1} \wedge t=\bar{\tau}\right\}}\left(a_{1} V_{1}^{0}\left(X_{\bar{\tau}}^{\bar{L}}\right)+a_{2} V_{2}^{0}\left(Y_{\bar{\tau}}^{\bar{L}}\right)\right)\right) \\
\geq \quad & E_{x, y}\left(a_{1} \int_{0}^{\tau_{1} \wedge t} e^{-\delta s} l_{1} d s+a_{2} \int_{0}^{\tau_{1} \wedge t} e^{-\delta s} l_{2} d s\right) \\
& +E_{x, y}\left(e^{-\delta(\tau \wedge t)} \varphi\left(X_{\tau \wedge t}^{\bar{L}}, Y_{\tau \wedge t}^{\bar{L}} I_{\left\{\tau_{1} \wedge t<\bar{\tau}\right\}}\right)\right. \\
& +E_{x, y}\left(e^{-\delta \bar{\tau}} I_{\left\{\tau_{1} \wedge t=\bar{\tau}\right\}}\left(a_{1} V_{1}^{0}\left(X_{\frac{\bar{L}}{\bar{L}}}\right)+a_{2} V_{2}^{0}\left(Y_{\bar{\tau}}^{\bar{L}}\right)\right)\right) .
\end{aligned}
$$

We can write

$$
\begin{aligned}
E_{x, y} & \left(e^{-\delta(\tau \wedge t)} \varphi\left(X_{\tau \wedge t}^{\bar{L}}, Y_{\tau \wedge t}^{\bar{L}}\right) I_{\left\{\tau_{1} \wedge t<\bar{\tau}\right\}}\right) \\
= & E_{x, y}\left(I_{\left\{t<\tau_{1}\right\}} e^{-\delta t} \varphi\left(X_{\tau_{1} \wedge t}^{\bar{L}}, Y_{\tau_{1} \wedge t}^{\bar{L}}\right)\right) \\
& +E_{x, y}\left(I_{\left\{\tau_{1}=\tau_{1} \wedge t<\bar{\tau} \text { and } \tau_{1}=\tau_{1}^{1}\right\}} e^{-\delta \tau_{1}^{1}} \varphi\left(X_{\tau_{1}^{1}}^{\bar{L}}, Y_{\tau_{1}^{1}}^{\bar{L}}\right)\right) \\
& +E_{x, y}\left(I_{\left\{\tau_{1}=\tau_{1} \wedge t<\bar{\tau} \text { and } \tau_{1}=\tau_{1}^{2}\right\}} e^{-\delta \tau_{1}^{2}} \varphi\left(X_{\tau_{1}^{2}}^{\bar{L}}, Y_{\tau_{1}^{2}}^{\bar{L}}\right)\right) .
\end{aligned}
$$

So we obtain 


$$
\begin{aligned}
& 0 \geq \lim _{t \rightarrow 0^{+}} E_{x, y}\left(\frac{a_{1} \int_{0}^{\tau_{1} \wedge t} e^{-\delta s} l_{1} d s+a_{2} \int_{0}^{\tau_{1} \wedge t} e^{-\delta s} l_{2} d s}{t}\right) \\
& +\lim _{t \rightarrow 0^{+}} \frac{e^{-(\lambda+\delta) t} \varphi\left(x+\left(p_{1}-l_{1}\right) t, y+\left(p_{2}-l_{2}\right) t\right)-\varphi(x, y)}{t} \\
& +\lim _{t \rightarrow 0^{+}} E_{x, y}\left(\frac{I_{\left\{\tau_{1}=\tau_{1} \wedge t<\bar{\tau} \text { and } \tau_{1}=\tau_{1}^{1}\right\}} e^{-\delta \tau_{1}^{1} \varphi\left(X_{\tau_{1}^{1}}^{\bar{L}}, Y_{\tau_{1}^{1}}^{\bar{L}}\right)}}{t}\right) \\
& +\lim _{t \rightarrow 0^{+}} E_{x, y}\left(\frac{I_{\left\{\tau_{1}=\tau_{1} \wedge t<\bar{\tau} \text { and } \tau_{1}=\tau_{1}^{2}\right\}} e^{-\delta \tau_{1}^{2} \varphi\left(X_{\tau_{1}^{2}}^{\bar{L}}, Y_{\tau_{1}^{2}}^{\bar{L}}\right)}}{t}\right) \\
& +\lim _{t \rightarrow 0^{+}} E_{x, y}\left(\frac{e^{-\delta \bar{\tau}} I_{\left\{\tau_{1} \wedge t=\bar{\tau}\right\}}\left(a_{1} V_{1}^{0}\left(X \frac{\bar{L}}{\tau}\right)+a_{2} V_{2}^{0}\left(Y_{\bar{\tau}}^{\bar{L}}\right)\right)}{t}\right) \\
& =a_{1} l_{1}+a_{2} l_{2}-(\delta+\lambda) \varphi(x, y) \\
& +\left(p_{1}-l_{1}\right) \varphi_{x}(x, y)+\left(p_{2}-l_{2}\right) \varphi_{y}(x, y)+\mathcal{I}(\varphi)(x, y) \\
& +U(x, y) \text {. }
\end{aligned}
$$

Therefore,

$$
0 \geq \mathcal{L}(\varphi)(x, y)+l_{1}\left(a_{1}-\varphi_{x}(x, y)\right)+l_{2}\left(a_{2}-\varphi_{y}(x, y)\right) .
$$

Taking $l_{1}=l_{2}=0, l_{1} \rightarrow \infty$ with $l_{2}=0$, and $l_{2} \rightarrow \infty$ with $l_{1}=0$, we obtain

$$
\max \left\{\mathcal{L}(\varphi)(x, y), a_{1}-\varphi_{x}(x, y), a_{2}-\varphi_{y}(x, y)\right\} \leq 0 . \square
$$

Proposition $7 V$ is a viscosity subsolution of the HJB equation (8).

Proof. Arguing by contradiction, we assume that $V$ is not a subsolution of (8) at $\left(x_{0}, y_{0}\right)$ with $x_{0}>0$ and $y_{0}>0$. With a similar proof to the one of Proposition 3.1 of Azcue and Muler (2014), but extending the definitions to two variables, we first show that there exist $\varepsilon>0, h \in\left(0, \min \left\{x_{0} / 2, y_{0} / 2\right\}\right)$ and a continuously differentiable function $\psi: \mathbf{R}_{+}^{2} \rightarrow \mathbf{R}$ such that $\psi$ is a test function for the subsolution of Equation (8) at $\left(x_{0}, y_{0}\right)$ and satisfies

$$
\psi_{x}(x, y) \geq a_{1}, \psi_{y}(x, y) \geq a_{2}
$$

for $(x, y) \in\left[0, x_{0}+h\right] \times\left[0, y_{0}+h\right]$,

$$
\mathcal{L}(\psi)(x, y) \leq-2 \varepsilon \delta
$$

for $(x, y) \in\left[x_{0}-h, x_{0}+h\right] \times\left[y_{0}-h, y_{0}+h\right]$, and

$$
V(x, y) \leq \psi(x, y)-2 \varepsilon
$$

for $(x, y) \in \mathbf{R}_{+}^{2} \backslash\left(x_{0}-h / 2, x_{0}+h / 2\right) \times\left(y_{0}-h / 2, y_{0}+h / 2\right)$.

Since $\psi$ is continuously differentiable, we can find a positive constant $C$ such that

$$
\mathcal{L}(\psi)(x, y) \leq C
$$

for all $(x, y) \in\left[0, x_{0}+2 h\right] \times\left[0, y_{0}+2 h\right]$.

Consider

$$
0<\theta<\left\{\frac{h}{2 \max \left\{p_{1}, p_{2}\right\}}, \frac{\lambda}{4 \delta(\delta+\lambda)}, \frac{\varepsilon \lambda}{2 C(\delta+\lambda)}\right\},
$$

and let us take any admissible strategy $\bar{L} \in \Pi_{x_{0}, y_{0}}$. Consider the corresponding controlled risk process $\left(X_{t}, Y_{t}\right)$ starting at $\left(x_{0}, y_{0}\right)$, and define the stopping times

$$
\begin{aligned}
\tau^{b}=\inf \{t>0: & \left.\left(X_{t}, Y_{t}\right) \in \partial\left(\left[x_{0}-h, x_{0}+h\right] \times\left[y_{0}-h, y_{0}+h\right]\right)\right\}, \\
\underline{\tau}=\inf \{t>0: & \left.\left(X_{t}, Y_{t}\right) \in \mathbf{R}_{+}^{2}-\left[x_{0}-h, x_{0}+h\right] \times\left[y_{0}-h, y_{0}+h\right]\right\}
\end{aligned}
$$


and $\tau^{*}=\tau^{b} \wedge(\underline{\tau}+\theta) \wedge \bar{\tau}$. Note that $\tau^{*}$ is finite for $h$ small enough and that it is necessary to introduce $\theta$ because before a lump sum dividend payment, $\left(X_{\underline{\tau}}, Y_{\underline{\tau}}\right)$ can be in $\left[x_{0}-h, x_{0}+\right.$ $h] \times\left[y_{0}-h, y_{0}+h\right]$ and $\left(X_{\underline{\tau}}, Y_{\underline{\tau}}\right) \in \mathbf{R}_{+}^{2}-\left[x_{0}-h, x_{0}+h\right] \times\left[y_{0}-h, y_{0}+h\right]$.

Let us show that

$$
V\left(X_{\tau^{*}}, Y_{\tau^{*}}\right) \leq \psi\left(X_{\tau^{*}}, Y_{\tau^{*}}\right)-2 \varepsilon
$$

if $\tau^{*}=\tau^{b} \wedge(\underline{\tau}+\theta)<\bar{\tau}$. There are two possibilities:

(1) If $\tau^{*}=\tau^{b},\left(X_{\tau^{*}}, Y_{\tau^{*}}\right) \in \partial\left(\left[x_{0}-h, x_{0}+h\right] \times\left[y_{0}-h, y_{0}+h\right]\right)$ and so, from (13), we obtain $V\left(X_{\tau^{*}}, Y_{\tau^{*}}\right) \leq \psi\left(X_{\tau^{*}}, Y_{\tau^{*}}\right)-2 \varepsilon$,

(2) If $\tau^{*}=\underline{\tau}+\theta$, the distance from $\left(X_{\tau^{*}}, Y_{\tau^{*}}\right)$ to $\left(x_{0}, y_{0}\right)$ is at least $h / 2 \geq h-\max \left\{p_{1}, p_{2}\right\} \theta$, so from (13), we get (15).

Note that $\left.\left.\left(X_{s^{-}}, Y_{s^{-}}\right) \in\left[0, x_{0}+h+p_{1} \theta\right] \times\left[0, y_{0}+h+p_{2} \theta\right)\right] \subset\left[0, x_{0}+2 h\right] \times\left[0, y_{0}+2 h\right)\right]$ for $s \leq \tau^{*}$, so we have that

$$
\mathcal{L}(\psi)\left(X_{s^{-}}, Y_{s^{-}}\right) \leq C \text { for } s \leq \tau^{*} .
$$

Since $L_{t}^{i}$, with $i=1,2$, is non-decreasing and left continuous, it can be written as

$$
L_{t}^{i}=\int_{0}^{t} d L_{s}^{i, c}+\sum_{\substack{X_{s} \neq X_{s} \\ s<t}}\left(L_{s}^{i}-L_{s}^{i}\right),
$$

where $L_{s}^{i, c}$ is a continuous and non-decreasing function. Since the function $\psi$ is continuously differentiable in $\mathbf{R}_{+}^{2}$, using the expression (16) and the change of variables formula for finite variation processes, we can write

$$
\begin{aligned}
& \psi\left(X_{\tau^{*}}, Y_{\tau^{*}}\right) e^{-\delta \tau^{*}}-\psi\left(x_{0}, y_{0}\right) \\
& =\quad \int_{0}^{\tau^{*}}\left(p_{1} \psi_{x}\left(X_{s^{-}}, Y_{s^{-}}\right)+p_{2} \psi_{y}\left(X_{s^{-}}, Y_{s^{-}}\right)\right) e^{-\delta s} d s \\
& \quad+\sum_{\substack{X_{s} \neq X_{s} \\
s \leq \tau^{*}}}\left(\psi\left(X_{s}, Y_{s}\right)-\psi\left(X_{s^{-}}, Y_{s^{-}}\right)\right) e^{-\delta s}+\sum_{\substack{Y_{s-1} \neq Y_{s} \\
s \leq \tau^{*}}}\left(\psi\left(X_{s}, Y_{s}\right)-\psi\left(X_{s^{-}}, Y_{s^{-}}\right)\right) e^{-\delta s} \\
& \quad-\int_{0}^{\tau^{*}} \psi_{x}\left(X_{s^{-}}, Y_{s^{-}}\right) e^{-\delta s} d L_{s}^{1, c}+\sum_{\substack{X_{s+\neq} \neq X_{s} \\
s<\tau^{*}}}\left(\psi\left(X_{s^{+}}, Y_{s^{+}}\right)-\psi\left(X_{s}, Y_{s}\right)\right) e^{-\delta s} \\
& \quad-\int_{0}^{\tau^{*}} \psi_{y}\left(X_{s^{-}}, Y_{s^{-}}\right) e^{-\delta s} d L_{s}^{2, c}+\sum_{\substack{Y_{s+7} \neq Y_{s} \\
s<\tau^{*}}}\left(\psi\left(X_{s^{+}}, Y_{s^{+}}\right)-\psi\left(X_{s}, Y_{s}\right)\right) e^{-\delta s} \\
& \quad-\delta \int_{0}^{\tau^{*}} \psi\left(X_{s^{-}}, Y_{s^{-}}\right) e^{-\delta s} d s .
\end{aligned}
$$

Note that $\left(X_{s}, Y_{s}\right) \in \mathbf{R}_{+}^{2}$ for $s \leq \tau^{*}$ except in the case that $\tau^{*}=\bar{\tau}$, where $X_{\tau^{*}}+Y_{\tau^{*}}<0$. Here we are extending the definition of $\psi$ as

$$
\psi(x, y)=a_{1} V_{1}^{0}(x) I_{x \geq 0}+a_{2} V_{2}^{0}(y) I_{y \geq 0}
$$

for $x+y<0$. We have that $X_{s^{+}} \neq X_{s}$ only at the jumps of $L_{s}^{1}$, and in this case $X_{s^{+}}-X_{s}=$ $-\left(L_{s^{+}}^{1}-L_{s}^{1}\right)$. Since $\bar{L}$ is admissible we have that $X_{s^{+}}=X_{s}-\left(L_{s^{+}}^{1}-L_{s}^{1}\right) \geq 0$. We can write

$$
\begin{aligned}
& -\int_{0}^{\tau^{*}} \psi_{x}\left(X_{s^{-}}, Y_{s^{-}}\right) e^{-\delta s} d L_{s}^{1, c}+\sum_{\substack{X_{s}+\neq X_{s} \\
s<\tau^{*}}}\left(\psi\left(X_{s^{+}}, Y_{s^{+}}\right)-\psi\left(X_{s}, Y_{s}\right)\right) e^{-\delta s} \\
& =-\int_{0}^{\tau^{*}} \psi_{x}\left(X_{s^{-}}, Y_{s^{-}}\right) e^{-\delta s} d L_{s}^{1, c}+\sum_{\substack{X_{s} \neq X_{s} \\
s<\tau^{*}}}\left(\int_{0}^{L_{s}^{1}+^{-L_{s}^{1}}} \psi_{x}\left(X_{s}-\alpha, Y_{s}\right) d \alpha\right) e^{-\delta s} \\
& \leq-\int_{0}^{\tau^{*}} a_{1} e^{-\delta s} d L_{s}^{1, c}-a_{1} \sum_{\substack{L_{s}^{1} \neq L_{s}^{1} \\
s<\tau^{*}}}\left(\int_{0}^{\left.L_{s}^{1}\right)^{-L_{s}^{1}}} d \alpha\right) e^{-\delta s} \\
& =-a_{1} \int_{0}^{\tau^{*}} e^{-\delta s} d L_{s}^{1} .
\end{aligned}
$$


Similarly,

$$
\begin{aligned}
& -\int_{0}^{\tau^{*}} \psi_{x}\left(X_{s^{-}}, Y_{s^{-}}\right) e^{-\delta s} d L_{s}^{2, c}+\sum_{\substack{X_{s}+\neq X_{s} \\
s<\tau^{*}}}\left(\psi\left(X_{s^{+}}, Y_{s^{+}}\right)-\psi\left(X_{s}, Y_{s}\right)\right) e^{-\delta s} \\
& =-\int_{0}^{\tau^{*}} \psi_{x}\left(X_{s^{-}}, Y_{s^{-}}\right) e^{-\delta s} d L_{s}^{2, c}-\sum_{\substack{L_{s+\neq L_{s}^{2}}^{2} \\
s<\tau^{*}}}\left(\int_{0}^{L_{s}^{2}{ }^{-L_{s}^{2}}} \psi_{x}\left(X_{s}, Y_{s}-\alpha\right) d \alpha\right) e^{-\delta s} \\
& \leq-a_{2} \int_{0}^{\tau^{*}} e^{-\delta s} d L_{s}^{2} .
\end{aligned}
$$

On the other hand, $X_{s} \neq X_{s^{-}}$only at the arrival of a claim for Company One, so

$$
\begin{aligned}
M_{t}^{1}= & \sum_{\substack{X_{s-1} \neq X_{s} \\
s \leq t}}\left(\psi\left(X_{s}, Y_{s}\right)-\psi\left(X_{s^{-}}, Y_{s^{-}}\right)\right) e^{-\delta s} \\
& -\lambda_{1} \int_{0}^{t} e^{-\delta s} \int_{0}^{X_{s^{-}}}\left(\psi\left(X_{s^{-}}-\alpha, Y_{s^{-}}\right)-\psi\left(X_{s^{-}}, Y_{s^{-}}\right)\right) d F^{1}(\alpha) d s \\
& -\lambda_{1} \int_{0}^{t} e^{-\delta s} \int_{X_{s^{-}}+Y_{s^{-}}}^{X_{s^{-}}}\left(\psi\left(0, X_{s^{-}}+Y_{s^{-}}-\alpha\right)-\psi\left(X_{s^{-}}, Y_{s^{-}}\right)\right) d F^{1}(\alpha) d s \\
& -\lambda_{1} \int_{0}^{t} e^{-\delta s} \int_{X_{s^{-}}+Y_{s^{-}}}^{\infty^{-}}\left(a_{2} V_{2}^{0}\left(Y_{s^{-}}\right)-\psi\left(X_{s^{-}}, Y_{s^{-}}\right)\right) d F^{1}(\alpha) d s
\end{aligned}
$$

is a martingale with zero expectation for $t \leq \bar{\tau}$. Analogously,

$$
\begin{aligned}
M_{t}^{2}= & \sum_{\substack{Y_{s-} \neq Y_{s} \\
s \leq t}}\left(\psi\left(X_{s}, Y_{s}\right)-\psi\left(X_{s^{-}}, Y_{s^{-}}\right)\right) e^{-\delta s} \\
& -\lambda_{2} \int_{0}^{t} e^{-\delta s} \int_{0}^{Y_{s^{-}}}\left(\psi\left(X_{s^{-}}, Y_{s^{-}}-\alpha\right)-\psi\left(X_{s^{-}}, Y_{s^{-}}\right)\right) d F^{2}(\alpha) d s \\
& -\lambda_{2} \int_{0}^{t} e^{-\delta s} \int_{X_{s^{-}}+Y_{s^{-}}}^{X_{s^{-}}}\left(\psi\left(X_{s^{-}}+Y_{s^{-}}-\alpha, 0\right)-\psi\left(X_{s^{-}}, Y_{s^{-}}\right)\right) d F^{2}(\alpha) d s \\
& -\lambda_{2} \int_{0}^{t} e^{-\delta s} \int_{X_{s^{-}}+Y_{s^{-}}}^{\infty^{-}}\left(a_{1} V_{1}^{0}\left(Y_{s^{-}}\right)-\psi\left(X_{s^{-}}, Y_{s^{-}}\right)\right) d F^{2}(\alpha) d s
\end{aligned}
$$

is also a martingale with zero expectation for $t \leq \bar{\tau}$. So we get

$$
\begin{array}{r}
\psi\left(X_{\tau^{*}}, Y_{\tau^{*}}\right) e^{-\delta \tau^{*}}-\psi\left(x_{0}, y_{0}\right) \leq \quad \int_{0}^{\tau^{*}} \mathcal{L}(\psi)\left(X_{s^{-}}, Y_{s^{-}}\right) e^{-\delta s}+M_{\tau^{*}}^{1}+M_{\tau^{*}}^{2} \\
-a_{1} \int_{0}^{\tau^{*}} e^{-\delta s} d L_{s}^{1}-a_{2} \int_{0}^{\tau^{*}} e^{-\delta s} d L_{s}^{2}
\end{array}
$$

Using the second inequality of (12), (14) and the definition of $\theta$ we get

$$
\begin{aligned}
\int_{0}^{\tau^{*}} \mathcal{L}(\psi)\left(X_{s^{-}}, Y_{s^{-}}\right) e^{-\delta s} d s & \leq \int_{0}^{\tau^{b} \wedge \underline{\tau} \wedge \bar{\tau}} \mathcal{L}(\psi)\left(X_{s^{-}}, Y_{s^{-}}\right) e^{-\delta s} d s+C \theta \\
& \leq-2 \varepsilon \delta \int_{0}^{\tau^{b} \wedge \underline{\tau} \wedge \bar{\tau}} e^{-\delta s} d s+C \theta \\
& \leq-2 \varepsilon \delta \int_{0}^{\tau^{*}} e^{-\delta s} d s+I_{\tau^{b} \wedge \underline{\tau} \wedge \bar{\tau}<\tau^{*}} 2 \varepsilon \delta \int_{\tau^{b} \wedge \underline{\tau} \wedge \bar{\tau}}^{\tau^{*}} e^{-\delta s} d s+C \theta \\
& \leq-2 \varepsilon\left(1-e^{-\delta \tau^{*}}\right)+\varepsilon \lambda /(\delta+\lambda) .
\end{aligned}
$$

From (23), Lemma 4 (15), (21) and (22), it follows that

$$
\begin{aligned}
& V\left(x_{0}, y_{0}\right) \\
&=\quad \sup _{\bar{L}}\left(E _ { x _ { 0 } , y _ { 0 } } \left(a_{1} \int_{0}^{\tau^{*}} e^{-\delta s} d L_{s}^{1}+a_{2} \int_{0}^{\tau^{*}} e^{-\delta s} d L_{s}^{2}+e^{-\delta \tau^{*}} V\left(X_{\tau^{*}}, Y_{\tau^{*}}\right) I_{\tau^{*}<\bar{\tau}}\right.\right. \\
&\left.\left.\quad+\left(a_{1} V_{1}^{0}\left(X_{\bar{\tau}}^{\bar{L}}\right)+a_{2} V_{2}^{0}\left(Y_{\bar{\tau}}^{\bar{L}}\right)\right) e^{-\delta \tau^{*}} I_{\tau^{*}=\bar{\tau}}\right)\right) \\
& \leq \quad \sup _{\bar{L}}\left(E _ { x _ { 0 } , y _ { 0 } } \left(a_{1} \int_{0}^{\tau^{*}} e^{-\delta s} d L_{s}^{1}+a_{2} \int_{0}^{\tau^{*}} e^{-\delta s} d L_{s}^{2}+e^{-\delta \tau^{*}}\left(\psi\left(X_{\tau^{*}}, Y_{\tau^{*}}\right)-2 \varepsilon\right) I_{\tau^{*}<\bar{\tau}}\right.\right. \\
&\left.\left.\quad+\left(a_{1} V_{1}^{0}\left(X_{\bar{\tau}}^{\bar{L}}\right)+a_{2} V_{2}^{0}\left(Y_{\bar{\tau}}^{\bar{L}}\right)\right) e^{-\delta \tau^{*}} I_{\tau^{*}=\bar{\tau}}\right)\right) \\
& \leq \sup _{\bar{L}} E_{x_{0}, y_{0}}\left(\int_{0}^{\tau^{*}} \mathcal{L}(\psi)\left(X_{s^{-}}, Y_{s^{-}}\right) e^{-\delta s} d s+M_{\tau^{*}}^{1}+M_{\tau^{*}}^{2}-2 \varepsilon e^{-\delta \tau^{*}} I_{\tau^{*}<\bar{\tau}}+\psi\left(x_{0}, y_{0}\right)\right) \\
& \leq \quad \psi\left(x_{0}, y_{0}\right)-\varepsilon \lambda /(\delta+\lambda) \\
&< \psi\left(x_{0}, y_{0}\right)
\end{aligned}
$$


and this contradicts the assumption that $V\left(x_{0}, y_{0}\right)=\psi\left(x_{0}, y_{0}\right)$.

From the above two propositions we get the following result.

Corollary $8 \mathrm{~V}$ is a viscosity solution of of the HJB equation (8).

\section{Smallest Viscosity Solution}

Let us prove now that the optimal value function $V$ is the smallest viscosity supersolution of (8).

We say that the function $u: \mathbf{R}_{+}^{2} \rightarrow \mathbf{R}$ satisfies the growth condition A.1, if

$$
u(x, y) \leq K+a_{1} x+a_{2} y \text { for all }(x, y) \in \mathbf{R}_{+}^{2} .
$$

The following Lemma is technical and will be used to prove Proposition 10 .

Lemma 9 Fix $x_{0}>0$ and $y_{0}>0$ and let $\bar{u}$ be a non-negative supersolution of (8) satisfying the growth condition A.1. We can find a sequence of positive functions $\bar{u}_{m}: \mathbf{R}_{+}^{2} \rightarrow \mathbf{R}$ such that:

(a) $\bar{u}_{m}$ is continuously differentiable.

(b) $\bar{u}_{m}$ satisfies the growth condition A.1.

(c) $p \leq p_{1} \bar{u}_{m, x}+p_{2} \bar{u}_{m, y} \leq(\delta+\lambda) \bar{u}_{m}$ in $\mathbf{R}_{+}^{2}$.

(d) $\bar{u}_{m} \searrow \bar{u}$ uniformly on compact sets in $\mathbf{R}_{+}^{2}$ and $\nabla \bar{u}_{m}$ converges to $\nabla \bar{u}$ a.e. in $\mathbf{R}_{+}^{2}$.

(e) There exists a sequence $c_{m}$ with $\lim _{m \rightarrow \infty} c_{m}=0$ such that

$$
\sup _{(x, y) \in A_{0}} \mathcal{L}\left(\bar{u}_{m}\right)(x, y) \leq c_{m} \text {, where } A_{0}=\left[0, x_{0}\right] \times\left[0, y_{0}\right] .
$$

Proof. The proof follows by standard convolution arguments and is the extension to two variables of Lemma 4.1 in Azcue an Muler 9 .

Proposition 10 The optimal value function $V$ is the smallest viscosity supersolution of (8) satisfying growth condition A.1.

Proof. Let $\bar{u}$ be a non-negative supersolution of (8) satisfying the growth condition A.1 and let $\bar{L} \in \Pi_{x, y}$; define $\left(X_{t}, Y_{t}\right)$ as the corresponding controlled risk process starting at $(x, y)$. Consider the function $\bar{u}_{m}$ of Lemma 9 in $\mathbf{R}_{+}^{2}$; we extend this function as

$$
\bar{u}_{m}(x, y)=a_{1} V_{1}^{0}(x) I_{x \geq 0}+a_{2} V_{2}^{0}(y) I_{y \geq 0} \text { for } x+y<0 .
$$

As in the proof of Proposition 7, we get

$$
\begin{aligned}
& \bar{u}_{m}\left(X_{t \wedge \bar{\tau}}, Y_{t \wedge \bar{\tau}}\right) e^{-\delta(t \wedge \bar{\tau})}-\bar{u}_{m}(x, y) \\
& \leq \int_{0}^{t \wedge \bar{\tau}} \mathcal{L}\left(\bar{u}_{m}\right)\left(X_{s^{-}}, Y_{s^{-}}\right) e^{-\delta s} d s-a_{1} \int_{0}^{t \wedge \bar{\tau}} e^{-\delta s} d L_{s}^{1}-a_{2} \int_{0}^{t \wedge \bar{\tau}} e^{-\delta s} d L_{s}^{2}+M_{t \wedge \bar{\tau}}^{1}+M_{t \wedge \bar{\tau}}^{2},
\end{aligned}
$$

where $M_{t}^{1}$ and $M_{t}^{2}$ are zero-expectation martingales. So we obtain that

$$
\begin{aligned}
\bar{u}_{m}\left(X_{t \wedge \bar{\tau}}, Y_{t \wedge \bar{\tau}}\right) e^{-\delta(t \wedge \bar{\tau})} I_{\bar{\tau}>t}-\bar{u}_{m}(x, y) \\
\leq \quad \int_{0}^{t \wedge \bar{\tau}} \mathcal{L}\left(\bar{u}_{m}\right)\left(X_{s^{-}}, Y_{s^{-}}\right) e^{-\delta s} d s+M_{t \wedge \bar{\tau}}^{1}+M_{t \wedge \bar{\tau}}^{2} \\
\quad-a_{1}\left(\int_{0}^{t \wedge \bar{\tau}} e^{-\delta s} d L_{s}^{1}+e^{-\delta(t \wedge \bar{\tau})} V_{1}^{0}\left(X_{t \wedge \bar{\tau}}\right) I_{\bar{\tau} \leq t}\right) \\
\quad-a_{2}\left(\int_{0}^{t \wedge \bar{\tau}} e^{-\delta s} d L_{s}^{2}+e^{-\delta(t \wedge \bar{\tau})} V_{2}^{0}\left(Y_{t \wedge \bar{\tau}}\right) I_{\bar{\tau} \leq t}\right)
\end{aligned}
$$

Using that both $L_{t}^{1}$ and $L_{t}^{2}$ are non-decreasing processes, from the monotone convergence theorem we get

$$
\begin{aligned}
& \lim _{t \rightarrow \infty}\left(E _ { x , y } \left(a_{1}\left(\int_{0}^{t \wedge \bar{\tau}} e^{-\delta s} d L_{s}^{1}+e^{-\delta(t \wedge \bar{\tau})} V_{1}^{0}\left(X_{t \wedge \bar{\tau}}\right) I_{\bar{\tau} \leq t}\right)\right.\right. \\
& \left.\left.+a_{2}\left(\int_{0}^{t \wedge \bar{\tau}} e^{-\delta s} d L_{s}^{2}+e^{-\delta(t \wedge \bar{\tau})} V_{2}^{0}\left(Y_{t \wedge \bar{\tau}}\right) I_{\bar{\tau} \leq t}\right)\right)\right) \\
& =V_{\bar{L}}(x, y) .
\end{aligned}
$$


From Lemma 9(c), we have

$$
-(\delta+\lambda) \bar{u}_{m}(x, y) \leq \mathcal{L}\left(\bar{u}_{m}\right)(x, y) \leq \lambda \bar{u}_{m}(x, y)+U(x, y) .
$$

But using Lemma 9(b) and the inequality $X_{s} \leq x+p_{1} s, Y_{s} \leq y+p_{2} s$ we get

$$
\bar{u}_{m}\left(X_{s}, Y_{s}\right) \leq K+a_{1} X_{s}+a_{2} Y_{s} \leq K+a_{1} x+a_{2} y+p s .
$$

So, using the bounded convergence theorem, we obtain

$$
\lim _{t \rightarrow \infty} E_{x, y}\left(\int_{0}^{t \wedge \bar{\tau}} \mathcal{L}\left(\bar{u}_{m}\right)\left(X_{s^{-}}, Y_{s^{-}}\right) e^{-\delta s} d s\right)=E_{x, y}\left(\int_{0}^{\bar{\tau}} \mathcal{L}\left(\bar{u}_{m}\right)\left(X_{s^{-}}, Y_{s^{-}}\right) e^{-\delta s} d s\right) .
$$

From (24), (25) and (27), we get

$$
\lim _{t \rightarrow \infty} E_{x, y}\left(\bar{u}_{m}\left(X_{t \wedge \bar{\tau}}, Y_{t \wedge \bar{\tau}}\right) e^{-\delta(t \wedge \bar{\tau})} I_{\bar{\tau}<t}\right)-\bar{u}_{m}(x, y) \leq E_{x, y}\left(\int_{0}^{\bar{\tau}} \mathcal{L}\left(\bar{u}_{m}\right)\left(X_{s^{-}}, Y_{s^{-}}\right) e^{-\delta s} d s\right)-V_{\bar{L}}(x, y) .
$$

Next, we show that

$$
\lim _{t \rightarrow \infty} E_{x, y}\left(\bar{u}_{m}\left(X_{t \wedge \bar{\tau}}, Y_{t \wedge \bar{\tau}}\right) e^{-\delta(t \wedge \bar{\tau})} I_{\bar{\tau}>t}\right)=0 .
$$

From (26), there exists a $\bar{K}$ such that

$$
E_{x, y}\left(\bar{u}_{m}\left(X_{t \wedge \bar{\tau}}, Y_{t \wedge \bar{\tau}}\right) e^{-\delta(t \wedge \bar{\tau})} I_{\bar{\tau}>t}\right) \leq\left(\bar{K}+a_{1} x+a_{2} y+p t\right) e^{-\delta t} .
$$

Since the last expression goes to 0 as $t$ goes to infinity, we have (29). Let us prove now that

$$
\limsup _{m \rightarrow \infty} E_{x, y}\left(\int_{0}^{\bar{\tau}} \mathcal{L}\left(\bar{u}_{m}\right)\left(X_{s^{-}}, Y_{s^{-}}\right) e^{-\delta s} d s\right) \leq 0 .
$$

Given any $\varepsilon>0$, we can find $T$ such that

$$
\int_{T}^{\infty} \mathcal{L}\left(\bar{u}_{m}\right)\left(X_{s^{-}}, Y_{s^{-}}\right) e^{-\delta s} d s<\frac{\varepsilon}{2}
$$

for any $m \geq 1$, as by virtue of (26), growth condition A.1, Lemma 9(b) and Lemma 9(c), and the growth property of $V_{1}^{0}$ and $V_{2}^{0}$, there exist positive constants $k_{0}, k_{1}, k_{2}$ and $\bar{p}$ such that

$$
\begin{aligned}
\mathcal{L}\left(\bar{u}_{m}\right)\left(X_{s^{-}}, Y_{s^{-}}\right) & \leq \lambda \bar{u}_{m}\left(X_{s^{-}}, Y_{s^{-}}\right)+U\left(X_{s^{-}}, Y_{s^{-}}\right) \\
& \leq k_{0}+k_{1} x+k_{2} y+\bar{p} s .
\end{aligned}
$$

Note that for $s \leq T, X_{s^{-}} \leq x_{0}:=x+p_{1} T, Y_{s^{-}} \leq y_{0}:=y+p_{2} T$. From Lemma 9(e) we can find $m_{0}$ large enough such that for any $m \geq m_{0}$

$$
\int_{0}^{T} \mathcal{L}\left(\bar{u}_{m}\right)\left(X_{s^{-}}, Y_{s^{-}}\right) e^{-\delta s} d s \leq c_{m} \int_{0}^{T} e^{-\delta s} d s \leq \frac{c_{m}}{\delta} \leq \frac{\varepsilon}{2}
$$

and so we get (30). Then, from (28) and using (29) and (30), we obtain

$$
\bar{u}(x, y)=\lim _{m \rightarrow \infty} \bar{u}_{m}(x, y) \geq V_{\bar{L}}(x, y) .
$$

Since $V$ is a viscosity solution of (8), the result follows.

From the previous proposition we can deduce the usual viscosity verification result.

Corollary 11 Consider a family of admissible strategies $\left\{\bar{L}^{x, y} \in \Pi_{x, y}:(x, y) \in \mathbf{R}_{+}^{2}\right\}$. If the function $V_{\bar{L}^{x, y}}(x, y)$ is a viscosity supersolution of (8) for all $(x, y) \in \mathbf{R}_{+}^{2}$, then $V_{\bar{L}^{x, y}}(x, y)$ is the optimal value function (4). 


\section{Iterative Approach}

In this section, we approximate the optimal value function $V$ defined in (4) by an increasing sequence of value functions of strategies which pay dividends (and collaborate if it is necessary) up to the $n$-th claim (regardless from which company) and then follow the take-the-moneyand-run strategy. Given initial surplus levels $(x, y)$, the take-the-money-and-run admissible strategy $\bar{L}^{0}$ pays immediately the entire surplus $x$ and $y$ as dividends (that is $X_{0^{+}}=Y_{0^{+}}=0$ ), and then pays the incoming premium as dividends until the first claim, where the company facing that claim gets ruined. Note that under this strategy the companies can not help each other.

Consider $\tau_{n}$ as the time of arrival of the $n$-th claim regardless from which company, that is the $n$-th point of the Poisson process $N_{t}=N_{t}^{1}+N_{t}^{2}$. We define the set $\Pi_{x, y}^{n}$ of all the admissible strategies in $\Pi_{x, y}$ which follow $\bar{L}^{0}$ right after $\tau_{n}$. Let us define

$$
V^{n}(x, y)=\sup _{\bar{L} \in \Pi_{x, y}^{n}} V_{\bar{L}}(x, y)
$$

for $n \geq 1$, we also define $V^{0}=V_{\bar{L}^{0}}$. We can write

$$
\begin{gathered}
V^{0}(x, y)=a_{1} x+a_{2} y+\frac{\lambda_{1}}{\delta+\lambda}\left(\frac{p}{\lambda}+a_{1} V_{2}^{0}(0)\right) \\
+\frac{\lambda_{2}}{\delta+\lambda}\left(\frac{p}{\lambda}+a_{2} V_{1}^{0}(0)\right) .
\end{gathered}
$$

Note that, for $n \geq 1$, we have

$$
V^{n}(x, y)=\sup _{\bar{L} \in \Pi_{x, y}} V_{\bar{L}}^{n}(x, y)
$$

where

$$
\begin{aligned}
V_{\bar{L}}^{n}(x, y)= & E_{x, y}\left(a_{1} \int_{0}^{\tau_{1}} e^{-\delta s} d L_{s}^{1}+a_{2} \int_{0}^{\tau_{1}} e^{-\delta s} d L_{s}^{2}+e^{-\delta \tau_{1}} V^{n-1}\left(X_{\tau_{1}}^{\bar{L}}, Y_{\tau_{1}}^{\bar{L}}\right) I_{\tau_{1}<\bar{\tau}}\right. \\
& \left.+e^{-\delta \bar{\tau}}\left(a_{1} V_{1}^{0}\left(X \frac{\bar{L}}{\tau}\right)+a_{2} V_{2}^{0}\left(Y_{\bar{\tau}}^{\bar{L}}\right)\right) I_{\tau_{1}=\bar{\tau}}\right)
\end{aligned}
$$

In this expression, we only consider the admissible strategy $\bar{L} \in \Pi_{x, y}$ for $t \leq \tau_{1}$. The following DPP holds.

Lemma 12 For any initial surplus $(x, y)$ in $\mathbf{R}_{+}^{2}$ and any stopping time $\tau$, we can write

$$
\begin{aligned}
V^{n}(x, y)= & \sup _{\bar{L} \in \Pi_{x, y}}\left(E _ { x , y } \left(a_{1} \int_{0}^{\tau_{1} \wedge \tau} e^{-\delta s} d L_{s}^{1}+a_{2} \int_{0}^{\tau_{1} \wedge \tau} e^{-\delta s} d L_{s}^{2}\right.\right. \\
& +e^{-\delta \tau} V^{n}\left(X_{\tau}^{\bar{L}}, Y_{\tau}^{\bar{L}}\right) I_{\tau<\tau_{1} \wedge \bar{\tau}}+e^{-\delta \tau_{1}} V^{n-1}\left(X_{\tau_{1}}^{\bar{L}}, Y_{\tau_{1}}^{\bar{L}}\right) I_{\tau_{1} \wedge \tau=\tau_{1}<\bar{\tau}} \\
& \left.\left.+e^{-\delta \bar{\tau}}\left(a_{1} V_{1}^{0}\left(X_{\bar{\tau}}^{\bar{L}}\right)+a_{2} V_{2}^{0}\left(Y_{\bar{\tau}}^{\bar{L}}\right)\right) I_{\tau_{1} \wedge \tau=\tau_{1}=\bar{\tau}}\right)\right) .
\end{aligned}
$$

Proposition 13 We have that $V^{1} \leq V^{2} \leq \ldots \leq V$.

Proof. We prove the result by induction:

(a) $V^{0} \leq V^{1}$, because the strategy $\bar{L}^{0} \in \Pi_{x, y}^{1}$.

(b) Assume that $V^{n-2} \leq V^{n-1}$. By (34), we have

$$
\begin{array}{rlr}
V^{n}(x, y) \geq & \sup _{\bar{L} \in \Pi_{x, y}} E_{x, y}\left(a_{1} \int_{0}^{\tau_{1}} e^{-\delta s} d L_{s}^{1}+a_{2} \int_{0}^{\tau_{1}} e^{-\delta s} d L_{s}^{2}+e^{-\delta \tau_{1}} V^{n-2}\left(X_{\tau_{1}}^{\bar{L}}, Y_{\tau_{1}}^{\bar{L}}\right) I_{\tau_{1}<\bar{\tau}}\right. \\
& \left.\quad+e^{-\delta \bar{\tau}}\left(a_{1} V_{1}^{0}\left(X_{\bar{\tau}}^{\bar{L}}\right)+a_{2} V_{2}^{0}\left(Y_{\bar{\tau}}^{\bar{L}}\right)\right) I_{\tau_{1}=\bar{\tau}}\right) & \\
= & \dot{V}^{n-1}(x, y) .
\end{array}
$$

The HJB equation for $V^{n}$ is given by 


$$
\max \left\{\mathcal{L}^{n}\left(V^{n}\right)(x, y), a_{1}-V_{x}^{n}(x, y), a_{2}-V_{y}^{n}(x, y)\right\}=0,
$$

where

$$
\mathcal{L}^{n}\left(V^{n}\right)(x, y)=p_{1} V_{x}^{n}(x, y)+p_{2} V_{y}^{n}(x, y)-(\delta+\lambda) V^{n}(x, y)+\mathcal{I}\left(V^{n-1}\right)(x, y)+U(x, y) .
$$

The following basic results about regularity and growth at infinity of $V^{n}, n \geq 1$ are similar to those of Lemmas 2 and

3.

Lemma 14 The optimal value function $V^{n}$ satisfies growth condition A.1, it is increasing and locally Lipschitz in $\mathbf{R}_{+}^{2}$ with

$$
\begin{aligned}
& a_{1} h \leq V^{n}(x+h, y)-V^{n}(x, y) \leq\left(e^{(\delta+\lambda) h / p_{1}}-1\right) V^{n}(x, y) \\
& a_{2} h \leq V^{n}(x, y+h)-V^{n}(x, y) \leq\left(e^{(\delta+\lambda) h / p_{2}}-1\right) V^{n}(x, y)
\end{aligned}
$$

for any $h>0$ and for any $(x, y)$ in $\mathbf{R}_{+}^{2}$.

In the next two propositions, we see that $V^{n}$ is a viscosity solution of the corresponding HJB equation.

Proposition $15 V^{n}$ is a viscosity supersolution of the HJB equation (36) for $x>0$ and $y>0$.

Proof. Similar to the one given in Proposition 6

Proposition $16 V^{n}$ is a viscosity subsolution of the corresponding HJB equation (36).

Proof. The proof of this proposition is similar to the one of Proposition 7 but using as martingales with zero expectation

$$
\begin{aligned}
M_{t}^{3}= & \sum_{\substack{X_{s^{-} \neq X_{s}} \\
s \leq t}}\left(V^{n-1}\left(X_{s}, Y_{s}\right)-\psi\left(X_{s^{-}}, Y_{s^{-}}\right)\right) e^{-\delta s} \\
& -\lambda_{1} \int_{0}^{t} e^{-\delta s} \int_{0}^{X_{s^{-}}}\left(V^{n-1}\left(X_{s^{-}}-\alpha, Y_{s^{-}}\right)-\psi\left(X_{s^{-}}, Y_{s^{-}}\right)\right) d F^{1}(\alpha) d s \\
& -\lambda_{1} \int_{0}^{t} e^{-\delta s} \int_{X_{s^{-}}+Y_{s^{-}}}^{X_{s^{-1}}}\left(V^{n-1}\left(0, X_{s^{-}}+Y_{s^{-}}-\alpha\right)-\psi\left(X_{s^{-}}, Y_{s^{-}}\right)\right) d F^{1}(\alpha) d s \\
& -\lambda_{1} \int_{0}^{t} e^{-\delta s} \int_{X_{s^{-}}+Y_{s^{-}}}^{\infty}\left(a_{2} V_{2}^{0}\left(Y_{s^{-}}\right)-\psi\left(X_{s^{-}}, Y_{s^{-}}\right)\right) d F^{1}(\alpha) d s
\end{aligned}
$$

and

$$
\begin{aligned}
M_{t}^{4}= & \sum_{\substack{Y_{s-} \neq Y_{s} \\
s \leq t}}\left(V^{n-1}\left(X_{s}, Y_{s}\right)-\psi\left(X_{s^{-}}, Y_{s^{-}}\right)\right) e^{-\delta s} \\
& -\lambda_{2} \int_{0}^{t} e^{-\delta s} \int_{0}^{Y_{s^{-}}}\left(V^{n-1}\left(X_{s^{-}}, Y_{s^{-}}-\alpha\right)-\psi\left(X_{s^{-}}, Y_{s^{-}}\right)\right) d F^{2}(\alpha) d s \\
& -\lambda_{2} \int_{0}^{t} e^{-\delta s} \int_{X_{s-}}^{X_{s^{-}}+Y_{s^{-}}}\left(V^{n-1}\left(X_{s^{-}}+Y_{s^{-}}-\alpha, 0\right)-\psi\left(X_{s^{-}}, Y_{s^{-}}\right)\right) d F^{2}(\alpha) d s \\
& -\lambda_{2} \int_{0}^{t} e^{-\delta s} \int_{X_{s^{-}} \infty^{-}}^{\infty_{s^{-}}}\left(a_{1} V_{1}^{0}\left(Y_{s^{-}}\right)-\psi\left(X_{s^{-}}, Y_{s^{-}}\right)\right) d F^{2}(\alpha) d s .
\end{aligned}
$$

instead of the martingales $M_{t}^{1}$ and $M_{t}^{2}$ defined in (19) and (20) respectively.

In the next proposition we state that $V^{n}$ is the smallest viscosity solution of the corresponding HJB equation.

Proposition 17 The optimal value function $V^{n}$ is the smallest viscosity supersolution of (36) satisfying growth condition A.1. 
Proof. The proof of this proposition is similar to the one of Proposition [10 but using as martingales with zero expectation (38) and (39).

Remark 18 From the above proposition we deduce the usual viscosity verification result for the n-step: Consider a family of admissible strategies $\left\{\bar{L}^{x, y} \in \Pi_{x, y}:(x, y) \in \mathbf{R}_{+}^{2}\right\}$. If the function $V_{\bar{L}^{x, y}}^{n}(x, y)$ is a viscosity supersolution of (36) then $V_{\bar{L}^{x, y}}^{n}=V^{n}$.

Finally, we have the convergence result to the optimal value function (4).

Proposition $19 V^{n} \nearrow V$ as $n$ goes to infinity.

Proof. By Lemma 3 and Lemma 2 is increasing and satisfies property A.1, so there exists a $T>0$ such that

$$
e^{-\delta t} V\left(x+p_{1} t, y+p_{2} t\right)<\frac{\varepsilon}{3}
$$

for $t \geq T$. Let us define $\kappa=V\left(x+p_{1} T, y+p_{2} T\right)>0$ and take $n_{0}>0$ such that

$$
P\left(\tau_{n_{0}} \geq T\right) \geq 1-\frac{\varepsilon}{3 \kappa} \text {. }
$$

There exists an admissible strategy $\bar{L} \in \Pi_{x, y}$ such that

$$
V(x, y)-V_{\bar{L}}(x, y) \leq \frac{\varepsilon}{3} .
$$

We define the strategy $\bar{L}^{n_{0}} \in \Pi_{x, y}^{n_{0}}$ as $\bar{L}_{t}^{n_{0}}=\bar{L}_{t}$ for $t \leq \tau_{n_{0}} \wedge \bar{\tau}$ and $\bar{L}_{t}^{n_{0}}=\bar{L}_{t-\tau_{n_{0}}}^{0}$ for $t \geq \tau_{n_{0}}$ if $\tau_{n_{0}}<\bar{\tau}$. From (40), (41) and Lemma 3, we have

$$
\begin{aligned}
& V_{\bar{L}}(x, y)-V_{\bar{L}^{n_{0}}}(x, y) \\
& \leq E_{x, y}\left(a_{1}\left(\int_{\bar{\tau} \wedge \tau_{n_{0}}}^{\bar{\tau}} e^{-\delta s} d L_{s}^{1}+e^{-\delta \bar{\tau}} V_{1}^{0}(X \bar{\tau})\right)+a_{2}\left(\int_{\bar{\tau} \wedge \tau_{n_{0}}}^{\bar{\tau}} e^{-\delta s} d L_{s}^{2}+e^{-\delta \bar{\tau}} V_{2}^{0}\left(Y_{\bar{\tau}}^{\bar{L}}\right)\right)\right) \\
& \leq E_{x, y}\left(e^{-\delta\left(\bar{\tau} \wedge \tau_{n_{0}}\right)} V\left(X_{\bar{\tau} \wedge \tau_{n_{0}}}^{\bar{L}}, Y_{\bar{\tau} \wedge \tau_{n_{0}}}^{\bar{L}}\right)\right) \\
& \leq E_{x, y}\left(I_{\left\{\bar{\tau} \wedge \tau_{n_{0}} \geq T\right\}} e^{-\delta\left(\bar{\tau} \wedge \tau_{n_{0}}\right)} V\left(x+p_{1}\left(\bar{\tau} \wedge \tau_{n_{0}}\right), y+p_{2}\left(\bar{\tau} \wedge \tau_{n_{0}}\right)\right)\right. \\
& +E_{x, y}\left(I_{\left\{\tau_{n_{0}}<T\right\}} e^{-\delta\left(\bar{\tau} \wedge \tau_{n_{0}}\right)} V\left(x+p_{1}\left(\bar{\tau} \wedge \tau_{n_{0}}\right), y+p_{2}\left(\bar{\tau} \wedge \tau_{n_{0}}\right)\right)\right. \\
& \leq E_{x, y}\left(I_{\left\{\bar{\tau} \wedge \tau_{n_{0}} \geq T\right\}} e^{-\delta\left(\bar{\tau} \wedge \tau_{n_{0}}\right)} V\left(x+p_{1}\left(\bar{\tau} \wedge \tau_{n_{0}}\right), y+p_{2}\left(\bar{\tau} \wedge \tau_{n_{0}}\right)\right)\right)+\kappa P\left(\tau_{n_{0}}<T\right) \\
& \leq \frac{2 \varepsilon}{3} \text {. }
\end{aligned}
$$

Then we obtain from (42)

$$
V(x, y) \leq V_{\bar{L}}(x, y)+\frac{\varepsilon}{3} \leq V_{\bar{L}^{n_{0}}}(x)+\varepsilon \leq V^{n}(x, y)+\varepsilon
$$

for any $n \geq n_{0}$.

\section{$6 \quad$ Stationary dividend strategies}

As in the one-dimensional case (see for instance Azcue and Muler 9]) our aim is to find a stationary dividend strategy whose value function is the optimal value function $V$. A dividend strategy is stationary when the decision on the dividend payment depends on the current surplus only, and not on the full history of the controlled process; note that a stationary dividend strategy generates a family of admissible strategies $\left\{\bar{L}^{x, y} \in \Pi_{x, y}\right.$ for any $\left.(x, y) \in \mathbf{R}_{+}^{2}\right\}$.

If we assume that the optimal value function $V$ is differentiable, the form in which the optimal value function solves the HJB equation at any $(x, y) \in \mathbf{R}_{+}^{2}$ suggests how the dividends should be paid when the current surplus is $(x, y)$. There are only seven possibilities: 
(i) If the current surplus is in the open set

$$
\mathcal{C}^{*}=\left\{(x, y) \in \mathbf{R}_{+}^{2}: \mathcal{L}(V)(x, y)=0, V_{x}(x, y)>a_{1}, V_{y}(x, y)>a_{2}\right\},
$$

no dividends are paid. The set $\mathcal{C}^{*}$ is called the non-action set.

(ii) If the current surplus is in the open set

$$
\mathcal{B}_{1}^{*}=\left\{(x, y) \in \mathbf{R}_{+}^{2}: \mathcal{L}(V)(x, y)<0, V_{x}(x, y)=a_{1}, V_{y}(x, y)>a_{2}\right\},
$$

Company One pays a lump sum as dividends. This lump sum should be $\min \{b>0$ : $\left.(x-b, y) \notin \mathcal{B}_{1}^{*}\right\}$.

(iii) If the current surplus is in the open set

$$
\mathcal{B}_{2}^{*}=\left\{(x, y) \in \mathbf{R}_{+}^{2}: \mathcal{L}(V)(x, y)<0, V_{x}(x, y)>a_{1}, V_{y}(x, y)=a_{2}\right\}
$$

Company Two pays a lump sum as dividends. This lump sum should be $\min \{b>0$ : $\left.(x, y-b) \notin \mathcal{B}_{2}^{*}\right\}$.

(iv) If the current surplus is in the set

$$
\mathcal{B}_{0}^{*}=\left\{(x, y) \in \mathbf{R}_{+}^{2}: \mathcal{L}(V)(x, y)<0, V_{x}(x, y)=a_{1}, V_{y}(x, y)=a_{2}\right\},
$$

but not in the closure of $\mathcal{B}_{1}^{*} \cup \mathcal{B}_{2}^{*}$, both companies pay a lump sum as dividends.

(v) If the current surplus is in the closed set

$$
\mathcal{A}_{0}^{*}=\left\{(x, y) \in \mathbf{R}_{+}^{2}: \mathcal{L}(V)(x, y)=0, V_{x}(x, y)=a_{1}, V_{y}(x, y)=a_{2}\right\},
$$

both companies pay their incoming premiums as dividends.

(vi) If the current surplus is in the set

$$
\mathcal{A}_{1}^{*}=\left\{(x, y) \in \mathbf{R}_{+}^{2}: \mathcal{L}(V)(x, y)=0, V_{x}(x, y)=a_{1}, V_{y}(x, y)>a_{2}\right\},
$$

Company One pays dividends at some special rate so that the surplus remains in $\mathcal{A}_{1}^{*} \cup \mathcal{A}_{0}^{*}$.

(vii) If the current surplus is in the set

$$
\mathcal{A}_{2}^{*}=\left\{(x, y) \in \mathbf{R}_{+}^{2}: \mathcal{L}(V)(x, y)=0, V_{x}(x, y)>a_{1}, V_{y}(x, y)=a_{2}\right\},
$$

Company Two pays dividends at some special rate so that the surplus remains in $\mathcal{A}_{2}^{*} \cup \mathcal{A}_{0}^{*}$.

Note that if $V$ is a continuously differentiable solution of (8), then $\mathcal{A}^{*}=\mathcal{A}_{0}^{*} \cup \mathcal{A}_{1}^{*} \cup \mathcal{A}_{2}^{*}$ is closed, $\mathcal{B}^{*}=\mathcal{B}_{1}^{*} \cup \mathcal{B}_{2}^{*} \cup \mathcal{B}_{0}^{*}$ is open, and any segment which connect a point of $\mathcal{B}^{*}$ with a point of the open set $\mathcal{C}^{*}$ should contain a point of $\mathcal{A}^{*}$.

Remark 20 Let us consider the simplest case of identical and independent Cramér-Lundberg processes in (1); that is $p_{1}=p_{2}=p ; \lambda_{1}=\lambda_{2}$; and $U_{i}^{1}, U_{i}^{2}$ have the same distribution $F$. We also choose the dividends paid by both companies to be equally weighted, i.e. $a_{1}=a_{2}=1 / 2$. Under these assumptions, the optimal value function will be symmetric, that is $V(x, y)=$ $V(y, x)$, and so the sets introduced above satisfy the following properties: the line $y=x$ is an axis of symmetry of the sets $\mathcal{C}^{*}, \mathcal{B}_{0}^{*}$ and $\mathcal{A}_{0}^{*}$; the sets $\mathcal{B}_{1}^{*}$ and $\mathcal{A}_{1}^{*}$ are the reflection with respect to the line $y=x$ of the sets $\mathcal{B}_{2}^{*}$ and $\mathcal{A}_{2}^{*}$ respectively. 


\section{Curve strategies}

We introduce a family of stationary dividend strategies, called curve strategies, in which dividends are paid in the seven ways mentioned in the previous section, having a simple structure: here the boundary between the action and non-action region is given by a curve. These strategies can be seen as the natural analogues of the one-dimensional barrier strategies in this two-dimensional case.

It is reasonable to think that if the optimal strategy is a curve strategy it should satisfy the following properties: If $\left(x_{0}, y_{0}\right) \in \mathcal{B}_{1}^{*} \cup \mathcal{A}_{1}^{*}$ (that is only Company One pays dividends), then $\left(x, y_{0}\right)$ should be in $\mathcal{B}_{1}^{*}$ for all $x>x_{0}$; analogously if $\left(x_{0}, y_{0}\right) \in \mathcal{B}_{2}^{*} \cup \mathcal{A}_{2}^{*}$ (that is only Company Two pays dividends), then $\left(x_{0}, y\right)$ should be in $\mathcal{B}_{2}^{*}$ for all $y>y_{0}$. Finally, the set $\mathcal{C}^{*}$ should be bounded because, as in the one-dimensional case, the surplus of each company under the optimal strategy should be bounded for $t>0$.

Let us define the curve strategies satisfying the properties mentioned above. For these strategies, $\mathbf{R}_{+}^{2}$ is partitioned into seven sets $\mathcal{C}, \mathcal{A}_{0}, \mathcal{A}_{1}, \mathcal{A}_{2}, \mathcal{B}_{0}, \mathcal{B}_{1}$ and $\mathcal{B}_{2}$ where $\mathcal{A}=\mathcal{A}_{0} \cup$ $\mathcal{A}_{1} \cup \mathcal{A}_{2}$ is a curve which intersects both coordinate axes.

- $\mathcal{A}_{0}=\{(\bar{x}, \bar{y})\}$ with $(\bar{x}, \bar{y}) \in \mathbf{R}_{+}^{2}$. If the current surplus is $(\bar{x}, \bar{y})$, both companies pay their incoming premium as dividends. Let us call $\bar{u}$ the $x$-intercept and $\bar{v}$ the $y$-intercept of the line with slope $p_{2} / p_{1}$ passing through $(\bar{x}, \bar{y})$; let us denote $\mathcal{O}_{1}^{(\bar{x}, \bar{y})}$ and $\mathcal{O}_{2}^{(\bar{x}, \bar{y})}$ the regions in the first quadrant bounded above and below by this line, respectively.

- $\mathcal{B}_{0}=[\bar{x}, \infty) \times[\bar{y}, \infty)-\mathcal{A}_{0}$. If the current surplus is $(x, y) \in \mathcal{B}_{0}$, Company One and Company Two pay $x-\bar{x}$ and $y-\bar{y}$ as dividends, respectively.

- The set $\mathcal{A}_{1}$ is a curve in $\mathcal{O}_{1}^{(\bar{x}, \bar{y})}$ parametrized by

$$
\mathcal{A}_{1}=\left\{\left(u+\frac{p_{1}}{p_{2}} \xi_{1}(u), \xi_{1}(u)\right) \text { with } \bar{u}<u \leq M_{\xi_{1}}\right\}
$$

where $\xi_{1}:\left[\bar{u}, M_{\xi_{1}}\right] \rightarrow \mathbf{R}$ is a continuously differentiable function with $\xi_{1}(\bar{u})=\bar{y}$, $\xi_{1}\left(M_{\xi_{1}}\right)=0$ and negative derivative. If the current surplus $(x, y) \in \mathcal{A}_{1}$, Company Two does not pay dividends and Company One pays dividends at some special rate for which the bivariate surplus remains in the curve $\mathcal{A}_{1}$. By basic calculus, it can be shown that this rate is given by

$$
l_{1}(x, y)=-\frac{p_{2}}{\xi_{1}^{\prime}\left(x-\left(p_{1} / p_{2}\right) y\right)} .
$$

- The set $\mathcal{B}_{1}$ is the set to the right of $\mathcal{A}_{1}$ in $\mathcal{O}_{1}^{(\bar{x}, \bar{y})}$, that is

$$
\mathcal{B}_{1}=\left\{(x, y) \in \mathbf{R}_{+}^{2}: y \leq \bar{y} \text { and } x>\xi_{1}^{-1}(y)+\frac{p_{1}}{p_{2}} y\right\} .
$$

If the current surplus $(x, y) \in \mathcal{B}_{1}$, Company Two does not pay dividends and Company One pays the lump sum

$$
\min \left\{b>0:(x-b, y) \in \mathcal{A}_{1}\right\}=x-\left(p_{1} / p_{2}\right) y-\xi_{1}^{-1}(y) .
$$

- The sets $\mathcal{A}_{2}$ and $\mathcal{B}_{2}$ in $\mathcal{O}_{2}^{(\bar{x}, \bar{y})}$ are defined analogously to $\mathcal{A}_{1}$ and $\mathcal{B}_{1}$ with the roles of Company One and Two interchanged; that is

$$
\mathcal{A}_{2}=\left\{\left(\xi_{2}(v), v+\frac{p_{2}}{p_{1}} \xi_{2}(v)\right) \text { with } \bar{v}<v \leq M_{\xi_{2}}\right\},
$$

and

$$
\mathcal{B}_{2}=\left\{(x, y) \in \mathbf{R}_{+}^{2}: x \leq \bar{x} \text { and } y>\xi_{2}^{-1}(x)+\frac{p_{2}}{p_{1}} x\right\}
$$

where $\xi_{2}:\left[\bar{v}, M_{\xi_{2}}\right] \rightarrow \mathbf{R}$ is a continuously differentiable function with $\xi_{2}(\bar{v})=\bar{x}$, $\xi_{2}\left(M_{\xi_{2}}\right)=0$ and negative derivative. If the current surplus $(x, y) \in \mathcal{A}_{2}$, Company One does not pay dividends and Company Two pays dividends at some special rate for which the bivariate surplus remains in the curve $\mathcal{A}_{2}$. Here this rate is

$$
l_{2}(x, y)=-\frac{p_{1}}{\xi_{2}^{\prime}\left(y-\left(p_{2} / p_{1}\right) x\right)} .
$$


- If the current surplus $(x, y) \in \mathcal{B}_{2}$, Company One does not pay dividends and Company Two pays the lump sum

$$
\min \left\{b>0:(x, y-b) \in \mathcal{A}_{2}\right\}=y-\left(p_{2} / p_{1}\right) x-\xi_{2}^{-1}(x) .
$$

- The no-action region $\mathcal{C}$ is the open set delimited by the curve $\mathcal{A}$ and the axes. If the current surplus $(x, y) \in \mathcal{C}$, no dividends are paid.

The set partition of the curve strategy corresponding to $(\bar{x}, \bar{y})=(1,2)$ and the functions

$$
\xi_{1}(u)=\frac{2(u-4)(u-6)}{35} \text { for } u \in[-1,4] \text { and } \xi_{2}(v)=\frac{(u-3)(u-6)}{10} \text { for } v \in[1,3]
$$

is illustrated in Figure 7.1.

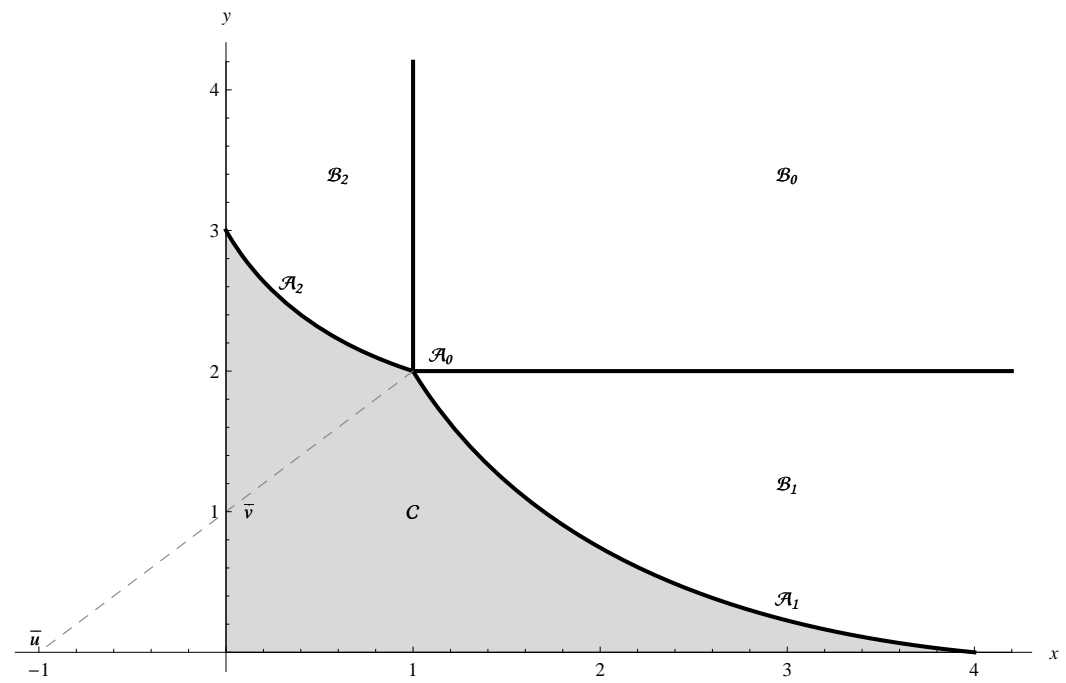

Fig. 7.1: Example of Curve Strategy

For any $w \in \mathbf{R}$, let us define the set

$$
\Phi^{w}=\left\{\xi:\left[w, M_{\xi}\right] \rightarrow \mathbf{R}_{+}, \xi\left(M_{\xi}\right)=0, M_{\xi} \geq 0, \xi^{\prime}<0 \text { and } \xi^{\prime} \text { continuous }\right\} .
$$

Note that the curve strategies depend only on the point $(\bar{x}, \bar{y}) \in \mathbf{R}_{+}^{2}$ and the functions $\xi_{1} \in \Phi^{\bar{u}}$ and $\xi_{2} \in \Phi^{\bar{v}}$, used in the parametrization of the curve $\mathcal{A}$. We associate to any $\bar{\xi}=\left((\bar{x}, \bar{y}), \xi_{1}, \xi_{2}\right)$ and any $(x, y) \in \mathbf{R}_{+}^{2}$ the admissible strategy $\bar{L}^{\bar{\xi}}=\left(L_{t}^{1, \bar{\xi}}, L_{t}^{2, \bar{\xi}}\right) \in \Pi_{x, y}$ Let us define the value function $V^{\bar{\xi}}$ of this curve strategy as

$$
V^{\bar{\xi}}(x, y)=V_{\bar{L}}(x, y) \text {. }
$$

We will look for $\bar{\xi}^{*}$ such that the associated value function $V^{\bar{\xi}^{*}}$ is the optimal value function defined in (4).

Remark 21 In the case that $\mathcal{A}$ is the segment $x+y=K$ for some $K>0$ in $\mathbf{R}_{+}^{2}$, the sum of the dividend rates paid by Company One and Two is $p_{1}+p_{2}$ for any current surplus in this line. The point $\mathcal{A}_{0}=(\bar{x}, K-\bar{x})$ indicates how this dividend payment is splitted among the two companies in $\mathcal{A}$ : At $\mathcal{A}_{0}$, Company One pays $p_{1}$ and Company Two pays $p_{2}$, to the right of this point $\left(\mathcal{A}_{1}\right)$ Company One pays the total rate $p_{1}+p_{2}$ and to the left of this point $\left(\mathcal{A}_{2}\right)$ it is Company Two which pays $p_{1}+p_{2}$. 


\section{Search for the Optimal Curve Strategy}

In order to find the optimal value function $V$ of the problem (4), we use the iterative approach introduced in Section 5 and Proposition [19] Our ultimate goal is to see whether the optimal value function $V$ is the value function of a curve strategy as defined in the previous section.

We first define an auxiliary function. For any $\bar{\xi}=\left((\bar{x}, \bar{y}), \xi_{1}, \xi_{2}\right)$, where $(\bar{x}, \bar{y}) \in \mathbf{R}_{+}^{2}$, $\xi_{1} \in \Phi^{\bar{u}}, \xi_{2} \in \Phi^{\bar{v}}$ and any continuous function $W_{0}: \mathbf{R}_{+}^{2} \rightarrow[0,+\infty)$, let

$$
\begin{aligned}
W^{\bar{\xi}}(x, y):= & E_{x, y}\left(\int_{0}^{\tau_{1}} e^{-\delta s}\left(a_{1} d L_{s}^{1, \bar{\xi}}+a_{2} d L_{s}^{2, \bar{\xi}}\right)+e^{-\delta \tau_{1}} W_{0}\left(X_{\tau_{1}}^{\bar{L}^{\xi}}, Y_{\tau_{1}}^{\bar{L}^{\xi}}\right) I_{\tau_{1}<\bar{\tau}}\right. \\
& \left.+e^{-\delta \bar{\tau}}\left(a_{1} V_{1}^{0}\left(X_{\bar{\tau}}^{\overline{L^{\xi}}}\right)+a_{2} V_{2}^{0}\left(Y_{\bar{\tau}}^{\bar{L}}\right)\right) I_{\tau_{1}=\bar{\tau}}\right) .
\end{aligned}
$$

If $W_{0}$ is the value function of a family of admissible strategies $\bar{L}=\left(\bar{L}_{x, y} \in \Pi_{x, y}\right)_{(x, y) \in \mathbf{R}_{+}^{2}}$, and $\mathcal{C}, \mathcal{A}_{0}, \mathcal{A}_{1}, \mathcal{A}_{2}, \mathcal{B}_{0}, \mathcal{B}_{1}$ and $\mathcal{B}_{2}$ are the sets associated to $\bar{\xi}$ (as defined in the previous section), then $W^{\bar{\xi}}$ would be the value function of the strategy which pays dividends according to the curve strategy $\bar{L} \bar{\xi}$ up to the first claim and according to $\bar{L}$ afterwards. We call this kind of strategy a one-step curve strategy.

Define

$$
H(x, y):=\mathcal{I}\left(W_{0}\right)(x, y)+U(x, y)
$$

In the next proposition, we find an explicit formula for the function $W^{\bar{\xi}}$ in terms of $W_{0}$ and $\xi_{1}$ for $(x, y) \in \mathcal{O}_{1}^{(\bar{x}, \bar{y})}$; the formula for the value function for $(x, y) \in \mathcal{O}_{2}^{(\bar{x}, \bar{y})}$ follows in an analogous way and depends only on $\xi_{2}$.

In order to obtain this formula, we use the fact that $W^{\bar{\xi}}$ satisfies the integro-differential equation $\mathcal{L}^{n}\left(W^{\bar{\xi}}\right)=0$ in $\mathcal{C} \cup \mathcal{A}$ and that $W_{x}^{\bar{\xi}}=a_{1}$ in $\mathcal{A}_{1} \cup \mathcal{A}_{0} \cup \mathcal{B}_{1} \cup \mathcal{B}$.

Proposition 22 Given $\bar{\xi}=\left((\bar{x}, \bar{y}), \xi_{1}, \xi_{2}\right)$ and a continuous function $W_{0}$, we have that

$$
\begin{aligned}
W^{\bar{\xi}}(x, y)= & e^{-(\delta+\lambda) \frac{\xi_{1}\left(x-\frac{p_{1}}{p_{2}} y\right)-y}{p_{2}}} k\left(x-\frac{p_{1}}{p_{2}} y\right) I_{\left\{(y-\bar{u}) \frac{p_{1}}{p_{2}} \leq x \leq \xi_{1}^{-1}(y)+\frac{p_{1}}{p_{2}} y, y \leq \bar{y}\right\}} \\
& +\left(\int_{0}^{\frac{\xi_{1}\left(x-\frac{p_{1}}{p_{2}} y\right)-y}{p_{2}}} e^{-(\delta+\lambda) w} H\left(x+p_{1} w, y+p_{2} w\right) d w\right) I_{\left\{(y-\bar{u}) \frac{p_{1}}{p_{2}} \leq x \leq \xi_{1}^{-1}(y)+\frac{p_{1}}{p_{2}} y, y \leq \bar{y}\right\}} \\
& +\left(a_{1}\left(x-\xi_{1}^{-1}(y)-\frac{p_{1}}{p_{2}} y\right)+k\left(\xi_{1}^{-1}(y)\right)\right) I_{\left\{x \geq \xi_{1}^{-1}(y)+\frac{p_{1}}{p_{2}} y, y \leq \bar{y}\right\}} \\
& +\left(a_{1}(x-\bar{x})+a_{2}(y-\bar{y})+k\left(\bar{x}-\frac{p_{1}}{p_{2}} \bar{y}\right)\right) I_{\{x \geq \bar{x}, y \geq \bar{y}\}},
\end{aligned}
$$

for $(x, y) \in \mathcal{O}_{1}^{(\bar{x}, \bar{y})}$, where $\bar{u}=\bar{x}-\frac{p_{1}}{p_{2}} \bar{y}$, the function $H$ is defined in 46) and

$$
\begin{aligned}
k(u)= & e^{(\delta+\lambda) \frac{\xi_{1}(u)-\xi_{1}(\bar{u})}{p_{2}}}\left(\frac{p}{\delta+\lambda}+\frac{1}{\delta+\lambda} H\left(\bar{u}+\frac{p_{1}}{p_{2}} \xi_{1}(\bar{u}), \xi_{1}(\bar{u})\right)\right) \\
& +e^{(\delta+\lambda) \frac{\xi_{1}(u)}{p_{2}}} a_{1} \int_{\bar{u}}^{u} e^{-(\delta+\lambda) \frac{\xi_{1}(w)}{p_{2}}} d w \\
& +\frac{e^{(\delta+\lambda) \frac{\xi_{1}(u)}{p_{2}}}}{p_{2}} \int_{\xi_{1}(u)}^{\xi_{1}(\bar{u})} H\left(\xi_{1}^{-1}(t)+\frac{p_{1}}{p_{2}} t, t\right) e^{-(\delta+\lambda) \frac{t}{p_{2}}} d t .
\end{aligned}
$$

Proof. Let us consider first an initial surplus $(x, y) \in \mathcal{C} \cap \mathcal{O}_{1}^{(\bar{x}, \bar{y})}$. By definition (45) we have that the controlled surplus process for $t<\tau_{1} \wedge h$ and $h>0$ small enough is given by

$$
\left(X_{t}, Y_{t}\right)=\left(x+p_{1} t, y+p_{2} t\right)
$$

So we have that 


$$
\begin{aligned}
W^{\bar{\xi}}(x, y)= & E_{x, y}\left(e^{-\delta t} W^{\bar{\xi}}\left(X_{t \wedge \tau_{1}}, Y_{t \wedge \tau_{1}}\right) I_{t \wedge \tau_{1}=t}+e^{-\delta \tau_{1}} W_{0}\left(X_{\tau_{1}}, Y_{\tau_{1}}\right) I_{t \wedge \tau_{1}=\tau_{1}<\bar{\tau}}\right. \\
& \left.+e^{-\delta \bar{\tau}}\left(a_{1} V_{1}^{0}\left(X_{\bar{\tau}}\right)+a_{2} V_{2}^{0}\left(Y_{\bar{\tau}}\right)\right) I_{t \wedge \tau_{1}=\tau_{1}=\bar{\tau}}\right) .
\end{aligned}
$$

We can write

$$
\begin{aligned}
E_{x, y} & \left.\left(e^{-\delta\left(\tau_{1} \wedge t\right)} I_{\left\{\tau_{1} \wedge t=t<\tau_{1}\right\}} W^{\bar{\xi}}\left(X_{\tau_{1} \wedge t}^{\bar{L}}, Y_{\tau_{1} \wedge t}^{\bar{L}}\right)+e^{-\delta\left(\tau_{1} \wedge t\right)} I_{\left\{\tau_{1} \wedge t=\tau_{1}<\bar{\tau}\right\}} W_{0}\left(X_{\tau_{1} \wedge t}^{\bar{L}}, Y_{\tau_{1} \wedge t}^{\bar{L}}\right)\right)\right) \\
= & E_{x, y}\left(e^{-\delta\left(\tau_{1} \wedge t\right)} I_{\tau_{1} \wedge t=t<\tau_{1}} W^{\bar{\xi}}\left(X_{\tau_{1} \wedge t}^{\bar{L}}, Y_{\tau_{1} \wedge t}^{\bar{L}}\right)\right) \\
& +E_{x, y}\left(I_{\left\{\tau_{1}=\tau_{1} \wedge t<\bar{\tau} \text { and } \tau_{1}=\tau_{1}^{1}\right\}} e^{-\delta \tau_{1}^{1}} W_{0}\left(X_{\tau_{1}^{1}}^{\bar{L}}, Y_{\tau_{1}^{1}}^{\bar{L}}\right)\right) \\
& +E_{x, y}\left(I_{\left\{\tau_{1}=\tau_{1} \wedge t<\bar{\tau} \text { and } \tau_{1}=\tau_{1}^{2}\right\}} e^{-\delta \tau_{1}^{2}} W_{0}\left(X_{\tau_{1}^{2}}^{\bar{L}}, Y_{\tau_{1}^{2}}^{\bar{L}}\right)\right),
\end{aligned}
$$

and so

$$
\lim _{t \rightarrow 0^{+}} \frac{e^{-(\lambda+\delta) t} W^{\bar{\xi}}\left(x+p_{1} t, y+p_{2} t\right)-W^{\bar{\xi}}(x, y)}{t}=-H(x, y) .
$$

Then $g(t)=W^{\bar{\xi}}\left(x+p_{1} t, y+p_{2} t\right)$ is continuous and differentiable as long as $\left(x+p_{1} t, y+p_{2} t\right) \in$ $\mathcal{C}$ with

$$
g^{\prime}(0)=(\lambda+\delta) W^{\bar{\xi}}(x, y)-H(x, y)
$$

Let us prove now that the function $W^{\bar{\xi}}$ is continuous in $\mathcal{A}_{1}$ and has a continuous derivative in the direction of this curve. In case $(x, y) \in \mathcal{A}_{1}$, we have that for $t<\tau_{1} \wedge h$ and $h>0$ small enough, the controlled surplus process is

$$
\left(X_{t}, Y_{t}\right)=\left(x+p_{1} t+\int_{0}^{t} \frac{p_{2}}{\xi_{1}^{\prime}\left(X_{s}-\left(p_{1} / p_{2}\right) Y_{s}\right)} d s, y+p_{2} t\right) \in \mathcal{A}_{1}
$$

By (45), we have that

$$
\begin{aligned}
W^{\bar{\xi}}(x, y) & =E_{x, y}\left(a_{1} \int_{0}^{\tau_{1} \wedge t} \frac{-p_{2}}{\xi_{1}^{\prime}\left(X_{s}-\left(p_{1} / p_{2}\right) Y_{s}\right)} e^{-\delta s} d s+e^{-\delta t} W^{\bar{\xi}}\left(X_{t \wedge \tau_{1}}, Y_{t \wedge \tau_{1}}\right) I_{t \wedge \tau_{1}=t}\right) \\
& +E_{x, y}\left(e^{-\delta \tau_{1}} W_{0}\left(X_{\tau_{1}}, Y_{\tau_{1}}\right) I_{t \wedge \tau_{1}=\tau_{1}<\bar{\tau}}+e^{-\delta \bar{\tau}}\left(a_{1} V_{1}^{0}\left(X_{\bar{\tau}}\right)+a_{2} V_{2}^{0}\left(Y_{\bar{\tau}}\right)\right) I_{t \wedge \tau_{1}=\tau_{1}=\bar{\tau}}\right) .
\end{aligned}
$$

Then, with an argument similar to the case of $\mathcal{C}$, we obtain for any $(x, y) \in \mathcal{A}_{1}$,

$$
\begin{aligned}
& \lim _{t \rightarrow 0} \frac{e^{-(\lambda+\delta) t} W^{\bar{\xi}}\left(x+p_{1} t+\int_{0}^{t} \frac{p_{2}}{\xi_{1}^{\prime}\left(X_{s}-\left(p_{1} / p_{2}\right) Y_{s}\right)} d s, y+p_{2} t\right)-W^{\bar{\xi}}(x, y)}{t} \\
& =-H(x, y)+\frac{a_{1} p_{2}}{\xi_{1}^{\prime}\left(x-\left(p_{1} / p_{2}\right) y\right)} .
\end{aligned}
$$

So

$$
g_{1}(t):=W^{\bar{\xi}}\left(x+p_{1} t+\int_{0}^{t} \frac{p_{2}}{\xi_{1}^{\prime}\left(X_{s}-\left(p_{1} / p_{2}\right) Y_{s}\right)} d s, y+p_{2} t\right)
$$

is continuous and differentiable at $t=0$ and satisfies

$$
g_{1}^{\prime}(0)=(\lambda+\delta) W^{\bar{\xi}}(x, y)-H(x, y)+\frac{a_{1} p_{2}}{\xi_{1}^{\prime}\left(x-\left(p_{1} / p_{2}\right) y\right)} .
$$

Since $\left(x+p_{1} t+\int_{0}^{t} p_{2} / \xi_{1}^{\prime}\left(X_{s}-\left(p_{1} / p_{2}\right) Y_{s}\right) d s, y+p_{2} t\right) \in \mathcal{A}_{1}$ for $t$ small enough, we have that

$$
\left(x+p_{1} t+\int_{0}^{t} \frac{p_{2}}{\xi_{1}^{\prime}\left(X_{s}-\left(p_{1} / p_{2}\right) Y_{s}\right)} d s, y+p_{2} t\right)=\left(u_{0}(t)+\frac{p_{1}}{p_{2}} \xi_{1}\left(u_{0}(t)\right), \xi_{1}\left(u_{0}(t)\right)\right)
$$


for $u_{0}(t):=x-\left(p_{1} / p_{2}\right) y+\int_{0}^{t} p_{2} / \xi_{1}^{\prime}\left(X_{s}-\left(p_{1} / p_{2}\right) Y_{s}\right) d s$; therefore

$$
W^{\bar{\xi}}\left(u_{0}(t)+\frac{p_{1}}{p_{2}} \xi_{1}\left(u_{0}(t)\right), \xi_{1}\left(u_{0}(t)\right)\right)=g_{1}(t) .
$$

Since $u_{0}^{\prime}(t)=p_{2} / \xi_{1}^{\prime}\left(X_{t}-\left(p_{1} / p_{2}\right) Y_{t}\right)$ is continuous and negative, $u_{0}^{-1}$ exists and is continuously differentiable, so

$$
k(u):=W^{\bar{\xi}}\left(u+\frac{p_{1}}{p_{2}} \xi_{1}(u), \xi_{1}(u)\right)=g_{1} \circ u_{0}^{-1}(u)
$$

is continuously differentiable.

Defining

$$
W(u, s):=W^{\bar{\xi}}\left(u+\frac{p_{1}}{p_{2}} \xi_{1}(u)-p_{1} s, \xi_{1}(u)-p_{2} s\right),
$$

we obtain for $\left(u+\left(p_{1} / p_{2}\right) \xi_{1}(u)-p_{1} s, \xi_{1}(u)-p_{2} s\right) \in \mathcal{C}$ that

$$
\begin{aligned}
& \widehat{\mathcal{L}}(W)(u, s) \\
& :=-W_{s}(u, s)-(\delta+\lambda) W(u, s)+H\left(u+\frac{p_{1}}{p_{2}} \xi_{1}(u)-p_{1} s, \xi_{1}(u)-p_{2} s\right)=0
\end{aligned}
$$

The equation $\widehat{\mathcal{L}}(W)(u, s)=0$ is a linear ODE in the variable $s$, so

$$
W(u, s) e^{(\delta+\lambda) s}-k(u)=\int_{0}^{s} e^{(\delta+\lambda) t} H\left(u+\frac{p_{1}}{p_{2}} \xi_{1}(u)-p_{1} t, \xi_{1}(u)-p_{2} t\right) d t
$$

therefore

$$
\begin{aligned}
& W^{\bar{\xi}}\left(u+\frac{p_{1}}{p_{2}} \xi_{1}(u)-p_{1} s, \xi_{1}(u)-p_{2} s\right) \\
& =e^{-(\delta+\lambda) s}\left(k(u)+\int_{0}^{s} e^{(\delta+\lambda) t} H\left(u+\frac{p_{1}}{p_{2}} \xi_{1}(u)-p_{1} t, \xi_{1}(u)-p_{2} t\right) d t\right),
\end{aligned}
$$

for $\bar{u} \leq u \leq M_{\xi_{1}}$ and $0 \leq s \leq \min \left\{\xi_{1}(u) / p_{2}, u / p_{1}+\xi_{1}(u) / p_{2}\right\}$. So $W^{\bar{\xi}}$ is continuously differentiable in the intersection of the set $\mathcal{C} \cup \mathcal{A}_{1}$ with $\mathcal{O}_{1}^{(\bar{x}, \bar{y})}$. We also have, from (47) and (48), that for any $(x, y) \in \mathcal{A}_{1}$,

$$
\begin{aligned}
& \lim _{t \rightarrow 0^{-}} \frac{W^{\bar{\xi}}\left(x+p_{1} t, y+p_{2} t\right)-W^{\bar{\xi}}(x, y)}{t} \\
& =\lim _{t \rightarrow 0^{+}} \frac{W^{\bar{\xi}}\left(x+p_{1} t+\frac{p_{2} t}{\xi_{1}^{\prime}\left(x-\frac{p_{1}}{p_{2}} y\right)}, y+p_{2} t\right)-W^{\bar{\xi}}(x, y)}{t}-a_{1} \frac{p_{2}}{\xi_{1}^{\prime}\left(x-\frac{p_{1}}{p_{2}} y\right)} .
\end{aligned}
$$

Then from

$$
\begin{aligned}
& p_{1} W_{x^{-}}^{\bar{\xi}}(x, y)+p_{2} W_{y^{-}}^{\bar{\xi}}(x, y) \\
& =\left(p_{1}+\frac{p_{2}}{\xi_{1}^{\prime}\left(x-\frac{p_{1}}{p_{2}} y\right)}\right) W_{x^{-}}^{\bar{\xi}}(x, y)+p_{2} W_{y^{-}}^{\bar{\xi}}(x, y)-a_{1} \frac{p_{2}}{\xi_{1}^{\prime}\left(x-\frac{p_{1}}{p_{2}} y\right)},
\end{aligned}
$$

we conclude that $W_{x^{-}}^{\bar{\xi}}(x, y)=a_{1}$.

By (49), and since $\left(u+\left(p_{1} / p_{2}\right) \xi_{1}(u), \xi_{1}(u)\right) \in \mathcal{A}_{1}$,

$$
\begin{aligned}
W_{x}^{\bar{\xi}}\left(u+\frac{p_{1}}{p_{2}} \xi_{1}(u), \xi_{1}(u)\right) & \left.=k^{\prime}(u)+\left(H\left(u+\frac{p_{1}}{p_{2}} \xi_{1}(u)\right), \xi_{1}(u)\right)-(\delta+\lambda) k(u)\right) \frac{\xi_{1}^{\prime}(u)}{p_{2}} \\
& =a_{1},
\end{aligned}
$$

and then 
$\left.k(u)=k(\bar{u}) e^{(\delta+\lambda) \frac{\xi_{1}(u)-\xi_{1}(\bar{u})}{p_{2}}}+\int_{\bar{u}}^{u}\left(a_{1}-H\left(w+\frac{p_{1}}{p_{2}} \xi_{1}(w)\right), \xi_{1}(w)\right) \frac{\xi_{1}^{\prime}(w)}{p_{2}}\right) e^{(\delta+\lambda) \frac{\xi_{1}(u)-\xi_{1}(w)}{p_{2}}} d w$.

At the point $\left(\bar{u}+\left(p_{1} / p_{2}\right) \xi_{1}(\bar{u}), \xi_{1}(\bar{u})\right) \in \mathcal{A}_{0}$ the dividend strategy consists of collecting all the incoming premium as dividends up to the time $\tau_{1}$, so

$$
k(\bar{u})=\frac{p}{\delta+\lambda}+\frac{1}{\delta+\lambda} H\left(\bar{u}+\frac{p_{1}}{p_{2}} \xi_{1}(\bar{u}), \xi_{1}(\bar{u})\right) .
$$

Then we have, from (50),

$$
\begin{aligned}
k(u)= & e^{(\delta+\lambda) \frac{\xi_{1}(u)-\xi_{1}(\bar{u})}{p_{2}}}\left(\frac{p}{\delta+\lambda}+\frac{1}{\delta+\lambda} H\left(\bar{u}+\frac{p_{1}}{p_{2}} \xi_{1}(\bar{u}), \xi_{1}(\bar{u})\right)\right) \\
& \left.+\int_{\bar{u}}^{u}\left(a_{1}-H\left(w+\frac{p_{1}}{p_{2}} \xi_{1}(w)\right), \xi_{1}(w)\right) \frac{\xi_{1}^{\prime}(w)}{p_{2}}\right) e^{(\delta+\lambda) \frac{\xi_{1}(u)-\xi_{1}(w)}{p_{2}}} d w .
\end{aligned}
$$

We conclude for (49) that for any $(x, y)$ in the intersection of the set $\mathcal{C}$ with $\mathcal{O}_{1}^{(\bar{x}, \bar{y})}$,

$$
\begin{aligned}
W^{\bar{\xi}}(x, y)= & e^{-(\delta+\lambda) \frac{\xi_{1}\left(x-\frac{p_{1}}{p_{2}} y\right)-y}{p_{2}} k\left(x-\frac{p_{1}}{p_{2}} y\right)} \\
& +\int_{0}^{\frac{\xi_{1}\left(x-\frac{p_{1}}{p_{2}} y\right)-y}{p_{2}}} e^{-(\delta+\lambda) w} H\left(x+p_{1} w, y+p_{2} w\right) d w
\end{aligned}
$$

which yields the result.

Remark 23 The formula of $W^{\bar{\xi}}$ in $\mathcal{O}_{2}^{(\bar{x}, \bar{y})}$ can be obtained using the formula given in Proposition [20 by interchanging the role of Company One and Company Two using that $W_{y}^{\bar{\xi}}=a_{2}$ in $\mathcal{A}_{2} \cup \mathcal{A}_{0} \cup \mathcal{B}_{2} \cup \mathcal{B}_{0}$. More precisely, if $(x, y) \in \mathcal{O}_{2}^{(\bar{x}, \bar{y})}$,

$$
\begin{aligned}
W^{\bar{\xi}}(x, y)= & e^{-(\delta+\lambda) \frac{\xi_{2}\left(y-\frac{p_{2}}{p_{1}} x\right)-x}{p_{1}}} \widetilde{k}\left(y-\frac{p_{2}}{p_{1}} x\right) I_{\left\{x \leq \bar{x}, y \leq \xi_{2}^{-1}(x)+\frac{p_{2}}{p_{1}} x\right\}} \\
& +\left(\int_{0}^{\frac{\xi_{2}\left(y-\frac{p_{2}}{p_{1}} x\right)-x}{p_{1}}} e^{-(\delta+\lambda) w} H\left(x+p_{1} w, y+p_{2} w\right) d w\right) I_{\left\{x \leq \bar{x}, y \leq \xi_{2}^{-1}(x)+\frac{p_{2}}{p_{1}} x\right\}} \\
& +\left(a_{2}\left(y-\xi_{2}^{-1}(x)-\frac{p_{2}}{p_{1}} x\right)+\widetilde{k}\left(\xi_{2}^{-1}(x)\right)\right) I_{\left\{x \leq \bar{x}, y \geq \xi_{2}^{-1}(x)+\frac{p_{2}}{p_{1}} x\right\}} \\
& +\left(a_{1}(x-\bar{x})+a_{2}(y-\bar{y})+\widetilde{k}\left(\bar{y}-\frac{p_{2}}{p_{1}} \bar{x}\right)\right) I_{\{x \geq \bar{x}, y \geq \bar{y}\}}
\end{aligned}
$$

where

$$
\begin{aligned}
\widetilde{k}(v)= & e^{(\delta+\lambda) \frac{\xi_{2}(v)-\xi_{2}(\bar{v})}{p_{1}}}\left(\frac{p}{\delta+\lambda}+\frac{1}{\delta+\lambda} H\left(\xi_{2}(\bar{v}), \bar{v}+\frac{p_{2}}{p_{1}} \xi_{2}(\bar{v})\right)\right) \\
& +e^{(\delta+\lambda) \frac{\xi_{2}(v)}{p_{1}}} a_{2} \int_{\bar{v}}^{v} e^{-(\delta+\lambda) \frac{\xi_{2}(w)}{p_{1}}} d w \\
& +\frac{e^{(\delta+\lambda) \frac{\xi_{2}(v)}{p_{1}}}}{p_{1}} \int_{\xi_{2}(v)}^{\xi_{2}(\bar{v})} H\left(t, \xi_{2}^{-1}(t)+\frac{p_{2}}{p_{1}} t\right) e^{-(\delta+\lambda) \frac{t}{p_{1}}} d t .
\end{aligned}
$$

From the formulas obtained in Proposition 22 and Remark 23 we obtain the following regularity result.

Proposition 24 If the function $H$ defined in (46) is continuously differentiable, then $W^{\bar{\xi}}$ is continuously differentiable in $\mathbf{R}_{+}^{2}$.

Proof. Since $\xi_{1}$ and $\xi_{2}$ are continuously differentiable, it is clear that $W^{\bar{\xi}}$ is continuously differentiable except possibly at the points of either the boundary of $\mathcal{B}_{0}$ or the segment

$$
\mathcal{S}=\left\{\left(x, \frac{p_{2}}{p_{1}}(x-\bar{x})+\bar{y}\right) \in \mathbf{R}_{+}^{2} \text { with } x \leq \bar{x}\right\} .
$$


After some easy calculations and using that $W^{\bar{\xi}}$ satisfies

$$
p-(\delta+\lambda) W^{\bar{\xi}}(\bar{x}, \bar{y})+H(\bar{x}, \bar{y})=0
$$

it can be seen that $W^{\bar{\xi}}$ is continuously differentiable in $\mathcal{S}$ with

$$
W_{x}^{\bar{\xi}}\left(x, \frac{p_{2}}{p_{1}}(x-\bar{x})+\bar{y}\right)=\int_{0}^{\frac{\bar{x}-x}{p_{1}}} e^{-(\delta+\lambda) w} H_{x}\left(x+p_{1} w, \frac{p_{2}}{p_{1}}(x-\bar{x})+\bar{y}+p_{2} w\right) d w+e^{-(\delta+\lambda) \frac{\bar{x}-x}{p_{1}}} a_{1}
$$

and

$$
W_{y}^{\bar{\xi}}\left(x, \frac{p_{2}}{p_{1}}(x-\bar{u})\right)=\int_{0}^{\frac{\bar{x}-x}{p_{1}}} e^{-(\delta+\lambda) w} H_{y}\left(x+p_{1} w, \frac{p_{2}}{p_{1}}(x-\bar{x})+\bar{y}+p_{2} w\right) d w+e^{-(\delta+\lambda) \frac{\bar{x}-x}{p_{1}}} a_{2} .
$$

Finally, the differentiability at the boundary of $\mathcal{B}_{0}$ follows from the differentiability of $W^{\bar{\xi}}$ at $(\bar{x}, \bar{y})$ of $\mathcal{S}$.

Let us define the set of functions

$$
\mathcal{M}=\left\{w: \mathbf{R}_{+}^{2} \rightarrow[0,+\infty) \text { continuous with } w(x, y)-a_{1} x-a_{2} y \text { bounded }\right\} .
$$

Proposition 25 The value function $V^{\bar{\xi}}$ of the curve strategy corresponding to $\bar{\xi}=\left((\bar{x}, \bar{y}), \xi_{1}, \xi_{2}\right)$ as defined in (44), satisfies the formulas given in Proposition 22 and Remark 23 replacing both $W_{0}$ and $W^{\bar{\xi}}$ by $V^{\bar{\xi}}$. Moreover, $V^{\bar{\xi}}$ is the unique function in $\mathcal{M}$ which satisfies this property.

Proof. $\mathcal{M}$ is a complete metric space with the distance $d\left(w_{1}, w_{2}\right)=\sup _{\mathbf{R}_{+}^{2}}\left|w_{1}-w_{2}\right|$. The operator $\mathcal{T}: \mathcal{M} \rightarrow \mathcal{M}$ defined as

$$
\begin{aligned}
\mathcal{T}(w)(x, y):=\quad & E_{x, y}\left(\int_{0}^{\tau_{1}} e^{-\delta s}\left(a_{1} d L_{s}^{1, \bar{\xi}}+a_{2} d L_{s}^{2, \bar{\xi}}\right)+e^{-\delta \tau_{1}} w\left(X_{\tau_{1}}^{\bar{L}^{\xi}}, Y_{\tau_{1}}^{\bar{L}^{\xi}}\right) I_{\tau_{1}<\bar{\tau}}\right. \\
& \left.+e^{-\delta \bar{\tau}}\left(a_{1} V_{1}^{0}\left(X_{\bar{\tau}}^{\bar{L}}\right)+a_{2} V_{2}^{0}\left(Y_{\bar{\tau}}^{\overline{L^{\xi}}}\right)\right) I_{\tau_{1}=\bar{\tau}}\right)
\end{aligned}
$$

is a contraction with contraction factor $\lambda /(\delta+\lambda)<1$. Then, there exists a unique fixed point and by definition (44), $\mathcal{T}\left(V^{\bar{\xi}}\right)=V^{\bar{\xi}}$. Taking in Proposition 22 and in Remark 23 the function $W_{0}$ as $V^{\bar{\xi}}$ we obtain from (45) that $V^{\bar{\xi}}=W^{\bar{\xi}}$ and so we get the result.

This last proposition gives a constructive way to obtain $V^{\bar{\xi}}$. Starting with $w_{0}(x, y)=$ $a_{1} x+a_{2} y \in \mathcal{M}$, we define iteratively $w_{n+1}=\mathcal{T}\left(w_{n}\right)$. Hence, $V^{\bar{\xi}}=\lim _{n \rightarrow \infty} w_{n}$. Note that at each step $w_{n+1}$ can be obtained from the formulas given in Proposition 22 and Remark 23 replacing $W_{0}$ by $w_{n}$.

Consider now the function $V^{n, \bar{\xi}}$ defined in (45) taking $W_{0}$ as the optimal value function $V^{n-1}$ corresponding to step $n-1$ in (33). We try to find $\bar{\xi}_{n}^{*}$, which maximizes $V^{n, \bar{\xi}}$ among all the possible $\bar{\xi}=\left((\bar{x}, \bar{y}), \xi_{1}, \xi_{2}\right)$. If the function $V^{n, \bar{\xi}_{n}^{*}}$ is a viscosity supersolution of (36), then by Remark 18, we would have that $V^{n, \bar{\xi}_{n}^{*}}=V^{n}$. In the case that one-step curve strategies corresponding to $\bar{\xi}_{n}^{*}$ exist for all $n \geq 1$, by Proposition 19, $V^{n, \bar{\xi}_{n}^{*}} \nearrow V$.

Let us call, as in (46),

$$
H_{n-1}(x, y):=\mathcal{I}\left(V^{n-1}\right)(x, y)+U(x, y) .
$$

In order to find the optimal one-step curve strategy corresponding to $\bar{\xi}_{n}^{*}=\left(\left(\bar{x}_{n}^{*}, \bar{y}_{n}^{*}\right), \xi_{1, n}^{*}, \xi_{2, n}^{*}\right)$, we look first for the optimal vertex $\left(\bar{x}_{n}^{*}, \bar{y}_{n}^{*}\right)$. By the formula given in Proposition 22

$$
V^{n, \bar{\xi}}(\bar{x}, \bar{y})=\frac{p}{\delta+\lambda}+\frac{H_{n-1}(\bar{x}, \bar{y})}{\delta+\lambda}
$$


and

$$
\begin{aligned}
& V^{n, \bar{\xi}}(x, y) \\
& =V^{n, \bar{\xi}}(\bar{x}, \bar{y})+a_{1}(x-\bar{x})+a_{2}(y-\bar{y}) .
\end{aligned}
$$

for $x$ and $y$ large enough. So

$$
\left(\bar{x}_{n}^{*}, \bar{y}_{n}^{*}\right)=\arg \max _{(\bar{x}, \bar{y}) \in \mathbf{R}_{+}^{2}} \frac{H_{n-1}(\bar{x}, \bar{y})}{\delta+\lambda}-a_{1} \bar{x}-a_{2} \bar{y} .
$$

If this maximum is attained at a critical point (assuming that $H_{n-1}$ is differentiable), we have that $\left(\bar{x}_{n}^{*}, \bar{y}_{n}^{*}\right)$ is a solution of

$$
\left\{\begin{array}{l}
\partial_{x} H_{n-1}(\bar{x}, \bar{y})=a_{1}(\delta+\lambda) \\
\partial_{y} H_{n-1}(\bar{x}, \bar{y})=a_{2}(\delta+\lambda) .
\end{array}\right.
$$

Let us call $\bar{u}_{n}^{*}=\bar{x}_{n}^{*}-\left(p_{1} / p_{2}\right) \bar{y}_{n}^{*}$ and $\bar{v}_{n}^{*}=\bar{y}_{n}^{*}-\left(p_{2} / p_{1}\right) \bar{x}_{n}^{*}$. Next, we use Calculus of Variations in order to find two curves $\xi_{1, n}^{*}$ and $\xi_{2, n}^{*}$ which maximize $V^{n, \bar{\xi}}(x, y)$, among all $\bar{\xi}=\left(\left(\bar{x}_{n}^{*}, \bar{y}_{n}^{*}\right), \xi_{1}, \xi_{2}\right)$ for $\xi_{1} \in \Phi^{\bar{u}_{n}^{*}}, \xi_{2} \in \Phi^{\bar{v}_{n}^{*}}$ and $(x, y)$ large enough. The two curves can be obtained separately and independently.

Proposition 26 Assume that $H_{n-1}$ is differentiable and that there exists $\bar{\xi}_{n}^{*}=\left(\left(\bar{x}_{n}^{*}, \bar{y}_{n}^{*}\right), \xi_{1, n}^{*}, \xi_{2, n}^{*}\right)$ where $\xi_{1, n}^{*} \in \Phi^{\bar{u}_{n}^{*}}$ and $\xi_{2, n}^{*} \in \Phi^{\bar{v}_{n}^{*}}$ such that $V^{n}=V^{n, \bar{\xi}_{n}^{*}}$. Then $\xi_{1, n}^{*}$ satisfies

$$
\partial_{x} H_{n-1}\left(u+\frac{p_{1}}{p_{2}} \xi_{1, n}^{*}(u), \xi_{1, n}^{*}(u)\right)=a_{1}(\delta+\lambda)
$$

for $\bar{u}_{n}^{*} \leq u \leq M_{\xi_{1, n}^{*}}$, and $\xi_{2, n}^{*}$ satisfies

$$
\partial_{y} H_{n-1}\left(\xi_{2, n}^{*}(v), v+\frac{p_{2}}{p_{1}} \xi_{2, n}^{*}(v)\right)=a_{2}(\delta+\lambda)
$$

for $\bar{v}_{n} \leq v \leq M_{\xi_{2, n}^{*}}$

Proof. We will prove this result for $\xi_{1, n}^{*}$, the proof for $\xi_{2, n}^{*}$ is analogous.

Given any $\xi_{1} \in \Phi^{\bar{u}_{n}^{*}}$, we have that

$$
V^{n, \bar{\xi}}\left(M_{\xi_{1}}, 0\right)+a_{1}\left(x-M_{\xi_{1}}\right),
$$

for $\bar{\xi}=\left(\left(\bar{x}_{n}^{*}, \bar{y}_{n}^{*}\right), \xi_{1}, \xi_{2}\right)$ and $x \geq M_{\xi_{1}}$. Then, if there exists $\xi_{1, n}^{*} \in \Phi^{\bar{u}_{n}^{*}}$ such that $V^{n}=V^{n, \bar{\xi}_{n}^{*}}$,

$$
V^{n, \bar{\xi}_{n}^{*}}\left(M_{\xi_{1, n}^{*}}, 0\right)-a_{1} M_{\xi_{1, n}^{*}}=\max _{\xi_{1} \in \Phi^{\bar{u}_{n}^{*}}}\left(V^{n, \bar{\xi}}\left(M_{\xi_{1}}, 0\right)-a_{1} M_{\xi_{1}}\right) .
$$

Consider non-negative test functions $\varsigma$ with $\varsigma\left(\bar{u}_{n}^{*}\right)=0$ and $\varsigma\left(M_{\xi_{1, n}^{*}}\right)=0$. We have that $\xi_{1, n}^{*}+\varepsilon \varsigma \in \Phi^{\bar{u}_{n}^{*}}$ for $\varepsilon$ small enough. Let us write,

$$
\xi_{\varepsilon}(u)=\xi_{1, n}^{*}(u)+\varepsilon \varsigma(u) .
$$

We have that $M_{\xi_{\varepsilon}}=M_{\xi_{1, n}^{*}}$ and then

$$
V^{n, \bar{\xi}_{n}^{*}}\left(M_{\xi_{1, n}^{*}}, 0\right)-a_{1} M_{\xi_{1, n}^{*}}=\max _{\varsigma}\left(V^{n, \bar{\xi}_{\varepsilon}}\left(M_{\xi_{1, n}^{*}}, 0\right)-a_{1} M_{\xi_{1, n}^{*}}\right),
$$

where $\bar{\xi}_{\varepsilon}=\left(\left(\bar{x}_{n}^{*}, \bar{y}_{n}^{*}\right), \xi_{\varepsilon}, \xi_{2}\right)$. Denote

$$
\vartheta(\varepsilon):=V^{n, \bar{\xi}_{\varepsilon}}\left(M_{\xi_{1, n}^{*}}, 0\right)-a_{1} M_{\xi_{1, n}^{*}} .
$$

We have that $\xi_{\varepsilon}\left(M_{\xi_{1, n}^{*}}\right)=\xi_{1, n}^{*}\left(M_{\xi_{1, n}^{*}}\right)=0$ and $\xi_{\varepsilon}\left(\bar{u}_{n}^{*}\right)=\xi_{1, n}^{*}\left(\bar{u}_{n}^{*}\right)$ so we can write by Proposition 22 


$$
\begin{aligned}
\vartheta(\varepsilon) & =e^{-\frac{(\delta+\lambda) \xi_{1, n}^{*}\left(\bar{u}_{n}^{*}\right)}{p_{2}}}\left(\frac{p}{\delta+\lambda}+\frac{1}{\delta+\lambda} H_{n-1}\left(\bar{u}_{n}^{*}+\frac{p_{1}}{p_{2}} \xi_{1, n}^{*}\left(\bar{u}_{n}^{*}\right), \xi_{1, n}^{*}\left(\bar{u}_{n}^{*}\right)\right)\right)-a_{1} \bar{u}_{n}^{*} \\
& +\int_{\bar{u}_{n}^{*}}^{M_{\xi_{1, n}^{*}}} a_{1}\left(e^{-\frac{(\delta+\lambda) \xi_{\varepsilon}(w)}{p_{2}}}-1\right) d w+\frac{1}{p_{2}} \int_{0}^{\xi_{1, n}^{*}\left(\bar{u}_{n}^{*}\right)} H_{n-1}\left(\xi_{\varepsilon}^{-1}(t)+\frac{p_{1}}{p_{2}} t, t\right) e^{-\frac{(\delta+\lambda) t}{p_{2}}} d t .
\end{aligned}
$$

Clearly,

$$
\begin{aligned}
0 & =\left.\frac{\vartheta(\varepsilon)}{\partial \varepsilon}\right|_{\varepsilon=0} \\
& =\frac{1}{p_{2}} \int_{\bar{u}_{n}^{*}}^{M_{\xi_{1, n}^{*}}}\left(\partial_{x} H_{n-1}\left(w+\frac{p_{1}}{p_{2}} \xi_{1, n}^{*}(w), \xi_{1, n}^{*}(w)\right)-a_{1}(\delta+\lambda)\right) e^{-(\delta+\lambda) \frac{\xi_{1, n}^{*}(w)}{p_{2}}} \varsigma(w) d w .
\end{aligned}
$$

So we obtain

$$
\left(\partial_{x} H_{n-1}\left(u+\frac{p_{1}}{p_{2}} \xi_{1, n}^{*}(u), \xi_{1, n}^{*}(u)\right)-a_{1}(\delta+\lambda)\right) e^{-(\delta+\lambda) \frac{\xi_{1, n}^{*}(u)}{p_{2}}}=0
$$

for all $\bar{u}_{n}^{*} \leq u \leq M_{\xi_{1, n}^{*}}$.

This last proposition gives us a constructive way to find the candidate for $V^{n}$ in the case that it comes from a one-step curve strategy. We find numerically, if it exists, the solution $z_{1}(u)$ of the equation

$$
\partial_{x} H_{n-1}\left(u+\frac{p_{1}}{p_{2}} z_{1}(u), z_{1}(u)\right)=a_{1}(\delta+\lambda)
$$

for $\bar{u}_{n}^{*} \leq u \leq \min \left\{u: z_{1}(u)=0\right\}$ and the solution $z_{2}(v)$ of the equation

$$
\partial_{y} H_{n-1}\left(z_{2}(v), v+\frac{p_{2}}{p_{1}} z_{2}(v)\right)=a_{2}(\delta+\lambda)
$$

for $\bar{v}_{n}^{*} \leq v \leq \min \left\{v: z_{2}(v)=0\right\}$. If $z_{1}(u)$ is in $\Phi^{\bar{u}_{n}^{*}}$ and $z_{2}(v)$ is in $\Phi^{\bar{v}_{n}}$, we define $\xi_{1, n}^{*}(u)=z_{1}(u)$ and $\xi_{2, n}^{*}(v)=z_{2}(v)$ and we obtain the value function $V^{n, \bar{\xi}_{n}^{*}}$ by the formula given in Proposition 22 this is our candidate for $V^{n}$. Afterwards, we check whether $V^{n, \bar{\xi}_{n}^{*}}$ is a viscosity supersolution of (36); if this is the case, then $V^{n}=V^{n, \bar{\xi}_{n}^{*}}$.

In the next Proposition we state some conditions under which the optimal strategy of (4) is a curve strategy. This result, together with Propositions 22 and 26 gives a way to find the optimal curve (if it exists). Let us first define a criterion of convergence for a sequence $\left(\bar{\xi}_{n}\right)_{n \geq 1}$ that will be used in the next proposition.

Definition 27 We say that $\bar{\xi}_{n}=\left(\left(\bar{x}_{n}, \bar{y}_{n}\right), \xi_{1, n}, \xi_{2, n}\right)$ converges to $\bar{\xi}=\left((\bar{x}, \bar{y}), \xi_{1}, \xi_{2}\right)$ if

$$
\begin{aligned}
& \lim _{n \rightarrow \infty}\left(\bar{x}_{n}, \bar{y}_{n}\right)=(\bar{x}, \bar{y}), \lim _{n \rightarrow \infty} M_{\xi_{i, n}} \rightarrow M_{\xi_{i}} \text { for } i=1,2, \\
& \lim _{n \rightarrow \infty\left[\bar{u}_{n}, M_{\xi_{1, n}}\right] \cap\left[\bar{u}, M_{\xi_{1}}\right]}\left|\xi_{1, n}(u)-\xi_{1}(u)\right|=0, \quad \lim _{n \rightarrow \infty\left[\bar{v}_{n}, M_{\xi_{2, n}}\right] \cap\left[\bar{v}, M_{\xi_{2}}\right]}\left|\xi_{2, n}(v)-\xi_{2}(v)\right|=0, \\
& \lim _{n \rightarrow \infty\left[0, \xi_{1, n}\left(\bar{u}_{n}\right)\right] \cap\left[0, \xi_{1}(\bar{u})\right]}\left|\xi_{1, n}^{-1}(w)-\xi_{1}^{-1}(w)\right|=0 \text { and } \lim _{n \rightarrow \infty\left[0, \xi_{2, n}\left(\bar{v}_{n}\right)\right] \cap\left[0, \xi_{2}(\bar{v})\right]}\left|\xi_{2, n}^{-1}(w)-\xi_{2}^{-1}(w)\right|=0 .
\end{aligned}
$$

Proposition 28 Assume that there exists a $\bar{\xi}_{n}^{*}$ such that $V^{n}=V^{n, \bar{\xi}_{n}^{*}}$ for all $n \geq 1$. If $\bar{\xi}_{n}^{*}$ converges to some $\bar{\xi}^{*}$ in the sense of Definition 27, then the optimal value function $V$ is the value function of the curve strategy $V^{\bar{\xi}^{*}}$ as defined in (44).

Proof. From Proposition 19 we have that $\lim _{n \rightarrow \infty} V^{n, \bar{\xi}_{n}^{*}}=V$. So replacing $W_{0}$ by $V^{n, \bar{\xi}_{n}^{*}}$ and $\bar{\xi}$ by $\bar{\xi}_{n}^{*}$ in the formulas given in Proposition 22 and Remark 23 and letting $n$ go to infinity, we obtain that $V$ satisfies the formulas given in Proposition 22 and Remark 23 replacing $W_{0}$ by $V$ and $\bar{\xi}$ by $\bar{\xi}^{*}$. Therefore, by Proposition 25 and Lemma 2, the functions $V$ and $V^{\bar{\xi}^{*}}$ coincide. 


\section{$9 \quad$ Numerical Example}

We present a numerical example in the symmetric and equally weighted case with an exponential claim size distribution. By Remark 20, we restrict the search of the optimal curve strategy to $\bar{\xi}=((\bar{x}, \bar{x}), \xi, \xi), \xi \in \Phi^{0}$. Using the formulas given in Propositions 22 and 26$]$ we obtain the functions $\xi_{n}^{*} \in \Phi^{0}$ and we check numerically that for $\bar{\xi}_{n}^{*}=\left(\left(\bar{x}_{n}^{*}, \bar{x}_{n}^{*}\right), \xi_{n}^{*}, \xi_{n}^{*}\right)$ the associated value function $V^{n, \bar{\xi}_{n}^{*}}$ is a viscosity solution of (36). We also obtain numerically the convergence of $\bar{\xi}_{n}^{*}$ to $\bar{\xi}$ according to Definition 27. Then, using Proposition 28, one can conclude that the optimal strategy is a curve strategy with curve $\xi^{*}$.

The numerical procedure was done with the Mathematica software and the calculation is quite time-consuming. The concrete chosen parameters are: exponential claim size distribution with parameter 3 , Poisson intensity $\lambda_{1}=\lambda_{2}=20 / 9$, premium rate $p_{1}=p_{2}=1$, and a discount factor $\delta=0.1$. In this numerical procedure we used step-size $\Delta x=\Delta y=0.002$ and iterated 60 times. The resulting optimal curve strategy is given in Figure 9.1, and $V(x, y)-(x+y) / 2($ the improvement of the optimal dividend strategy over paying out the initial capital immediately) is depicted as the upper curve in Figure 9.2.

We also compare for this numerical example the optimal value function $V(x, y)$ with the (comparably weighted) sum of the stand-alone value functions without collaboration:

$$
V_{S}(x, y)=\frac{V^{0}(x)+V^{0}(y)}{2}
$$

and with $V_{M}(x+y) / 2$, where $V_{M}$ is the optimal value function for the merger of the two companies. Figure 9.2 depicts the graphics of all three value functions $V(x, y), V_{S}(x, y)$ and $V_{M}(x+y) / 2$, each of them reduced by $(x+y) / 2$. The optimal merger strategy is barrier with barrier $b=2.77$. By Remark $1 V_{M}(x+y) / 2<V(x, y)$ for all $(x, y) \in \mathbf{R}_{+}^{2}$. One sees that whereas for the comparison between the stand-alone case and the merger the initial surplus levels matter (with the merger case being the lowest of the three value functions in $(0,0)$ ), the collaboration case outperforms not only the merger case but also the stand-alone one for all combinations of initial surplus levels (i.e. if one measures the overall dividend payments that can be achieved with either behavior, for this numerical example collaboration is always preferable). Hence we have here an instance where collaboration is beneficial not only for safety aspects, but also with respect to collective profitability.

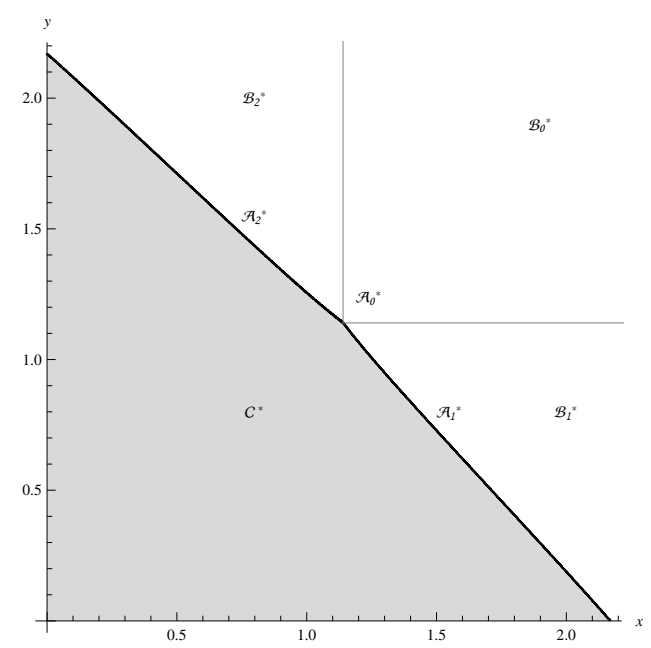

Figure 9.1: Optimal Curve Strategy 


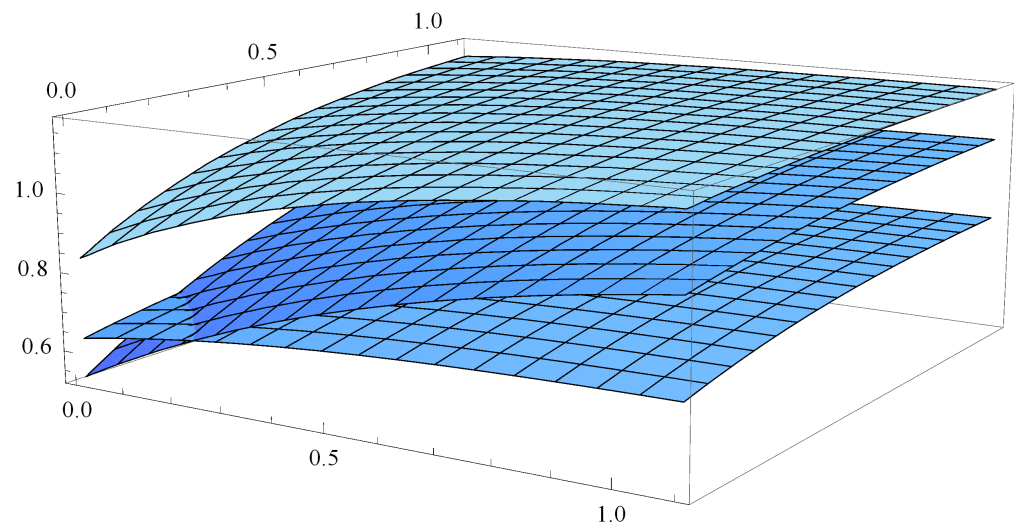

Figure 9.2: $V(x, y)-\frac{x+y}{2}$ vs. $V_{S}(x, y)-\frac{x+y}{2}$ vs. $\frac{V_{M}(x+y)}{2}-\frac{x+y}{2}$

\section{References}

[1] H. Albrecher and V. Lautscham. Dividends and the time of ruin under barrier strategies with a capital-exchange agreement. Preprint, University of Lausanne, 2014.

[2] H. Albrecher and S. Thonhauser. Optimality results for dividend problems in insurance. Rev. R. Acad. Cienc. Exactas Fís. Nat. Ser. A Math. RACSAM, 103(2):295-320, 2009.

[3] S. Asmussen and H. Albrecher. Ruin probabilities. Advanced Series on Statistical Science \& Applied Probability, 14. World Scientific Publishing Co. Pte. Ltd., Hackensack, NJ, second edition, 2010.

[4] B. Avanzi. Strategies for dividend distribution: a review. N. Am. Actuar. J., 13(2):217251, 2009.

[5] F. Avram, Z. Palmowski, and M. Pistorius. A two-dimensional ruin problem on the positive quadrant. Insurance Math. Econom., 42(1):227-234, 2008.

[6] F. Avram, Z. Palmowski, and M. R. Pistorius. Exit problem of a two-dimensional risk process from the quadrant: exact and asymptotic results. Ann. Appl. Probab., 18(6):2421$2449,2008$.

[7] P. Azcue and N. Muler. Optimal reinsurance and dividend distribution policies in the Cramér-Lundberg model. Math. Finance, 15(2):261-308, 2005.

[8] P. Azcue and N. Muler. Minimizing the ruin probability allowing investments in two assets: a two-dimensional problem. Math. Methods Oper. Res., 77(2):177-206, 2013.

[9] P. Azcue and N. Muler. Stochastic Optimization in Insurance: a Dynamic Programming Approach. Springer Briefs in Quantitative Finance. Springer, 2014.

[10] A. Badescu, L. Gong, and S. Lin. Optimal capital allocations for a bivariate risk process under a risk sharing strategy. Preprint, University of Toronto, 2015.

[11] S. Badila, O. Boxma, and J. Resing. Two parallel insurance lines with simultaneous arrivals and risks correlated with inter-arrival times. Insurance Math. Econom., 61:4861, 2015.

[12] M. G. Crandall and P.-L. Lions. Viscosity solutions of Hamilton-Jacobi equations. Trans. Amer. Math. Soc., 277(1):1-42, 1983.

[13] I. Czarna and Z. Palmowski. De Finetti's dividend problem and impulse control for a two-dimensional insurance risk process. Preprint, arXiv:0906.2100v3, 2011. 
[14] B. De Finetti. Su un' impostazione alternativa dell teoria collettiva del rischio. Transactions of the XVth congress of actuaries, (II):433-443, 1957.

[15] H. U. Gerber. Entscheidungskriterien fuer den zusammengesetzten Poisson-Prozess. Schweiz. Aktuarver. Mitt., (1):185-227, 1969.

[16] H. U. Gerber and E. S. W. Shiu. On the merger of two companies. N. Am. Actuar. J., 10(3):60-67, 2006.

[17] J. Ivanovs and O. Boxma. A bivariate risk model with mutual deficit coverage. Preprint, University of Lausanne, 2015. arXiv:1501.02927.

[18] N. Kulenko and H. Schmidli. Optimal dividend strategies in a Cramér-Lundberg model with capital injections. Insurance Math. Econom., 43(2):270-278, 2008.

[19] R. L. Loeffen and J.-F. Renaud. De Finetti's optimal dividends problem with an affine penalty function at ruin. Insurance Math. Econom., 46(1):98-108, 2010.

[20] R. Radner and L. Shepp. Risk vs. profit potential: a model for corporate strategy. Journal of Economic Dynamics and Control, 20:1373-1393, 1996.

[21] H. Schmidli. Stochastic control in insurance. Probability and its Applications (New York). Springer-Verlag London, Ltd., London, 2008.

[22] H. M. Soner. Optimal control with state-space constraint. I. SIAM J. Control Optim., 24(3):552-561, 1986.

[23] S. Thonhauser and H. Albrecher. Dividend maximization under consideration of the time value of ruin. Insurance Math. Econom., 41(1):163-184, 2007. 